\title{
NORMAL MODES FOR LARGE AMPLITUDE VIBRATION OF A CANTILEVER BEAM
}

\author{
Shang-Rou Hsieh, Steven W. Shaw and Christophe Pierre \\ Department of Mechanical Engineering and Applied Mechanics, The University of Michigan, \\ Ann Arbor, MI 48109-2125, U.S.A.
}

(Received 14 January 1993 ; in revised form 22 September 1993)

\begin{abstract}
We consider the non-linear normal modes for finite amplitude vibrations of a cantilever beam by using an extension of the invariant manifold techniques recently developed by the authors. Detailed results from two examples are presented. The first considers non-linearities which arise from finite deformations, including inertial non-linearities. In this case it is shown that if one simply accounts for the kinemalic effects of foreshortening, the linear modes of the cantilever beam work remarkably well for describing the actual non-linear mode shapes of the beam up to quite large amplitudes. A second example demonstrates the manner in which the method handles non-linearities in the boundary conditions, in this case, a non-linear spring attached at the free end of the beam.
\end{abstract}

\section{INTRODUCTION}

The concept of normal modes of motion (Courant and Hilbert, 1961) is well developed for a wide class of dynamical systems described by linear ordinary or partial differential equations with constant coefficients. A normal mode for a conservative, non-gyroscopic system can be defined as a motion for which the ratios amongst the generalized coordinates are independent of time. Such a normal mode motion is synchronous and constitutes a standing wave. Each normal mode has associated with it a mode shape, defined by an eigenfunction (or an eigenvector), and a corresponding natural frequency, defined by the associated eigenvalue. For non-conservative and/or gyroscopic systems, normal modes of motion also exist, although the situation is not so simple, since the modes are typically traveling waves. For nearly all such linear systems, the dynamics of an individual normal mode is described by a second-order linear model oscillator which is uncoupled from all other modal oscillators. Furthermore, a general motion can be written as a linear combination of these normal modes. For non-linear systems, such a principle of superposition does not hold. However, it is still possible to define normal modes for non-linear systems, and much work has been done in the case of systems with a finite number of degrees of freedom (Rosenberg, 1966). However, until very recently, a normal mode formulation for continuous, infinite degree of freedom non-linear systems has been lackling. The purpose of this paper is to use the methodology recently developed by Pierre and Shaw (1992) and Shaw and Pierre $(1993,1994)$ to investigate the non-linear normal modes of a common structural system - the cantilever beam with geometric non-linearities arising from large deformations. The work presented here also demonstrates how the method developed by Shaw and Pierre (1993) can be extended to handle non-linear inertia terms.

The main feature of the method presented by Pierre and Shaw (1991) and Shaw and Pierre $(1993,1994)$ and adopted here is that normal modes are described by two-dimensional invariant manifolds in the phase space of the non-linear system. These manifolds are simply curved surfaces, tangent to the eigenspaces of the linearized system, which can be defined for a wide class of problems, including those with damping and/or gyroscopic terms. The equations of motion restricted to these manifolds are then used to generate the non-linear oscillators which describe the dynamics of the non-linear normal modes. This method reduces to an equivalent of Rosenberg's method in the case of conservative systems.

Previous investigations on the topic of non-linear normal modes have been mostly limited to finite-dimensional conservative systems (Rosenberg, 1966; Rand, 1974; Vakakis, 1990). Only a few efforts related to non-linear continuous systems have appeared in the literature (Benamar et al., 1991 ; Bennouna and White, 1984; Szemplinska, 1990) and these are restricted to assumptions of standing-wave, time-harmonic motions of conservative 
systems. The methodology chosen here differs in that it may be applied to non-conservative and/or gyroscopic systems and, more importantly, it does not require one to assume a timeharmonic motion. Rather, the (amplitude-dependent) mode shapes are first determined, and then the dynamics of the normal mode are obtained from a non-linear oscillator equation. Moreover, the approach is based on a non-local formulation, and it does not require a series representation of the mode shapes at the outset of the solution procedure, as do the methods found in Benamar et al. (1991), Bennouna and White (1984) and Szemplinska (1990). However, it must be admitted that, for many problems, the only practical solution procedure is local in nature and is based on the form of an asymptotic series, but this assumption is by no means required at the outset of the procedure. In addition, the present methodology allows for the separation of the temporal and spatial behaviors in such a manner that the system changes its deflection shape in an amplitudedepcndent manner during a given motion. Previous methods fixed the deflection shape and allowed only for the amplitude to be time dependent. In summary, the methodology adopted here represents a formal, systematic means of defining and constructing normal modes for non-linear systems, and it is based on first principles from the theory of dynamical systems.

This paper is organized as follows. Section 2 provides a description of the general approach for the class of problems to be investigated. In the following two sections, two examples are used to demonstrate the technique. Section 3 describes the analysis and the results for the non-linear normal modes of large amplitude vibrations of a cantilever beam. The normal modes of a linear cantilever beam with a non-linear spring attached to its free ends are analyzed in Section 4. In Section 5, the convergence properties of the non-linear normal mode solutions are examined. The paper closes with a conclusion in Section 6.

\section{THE GENERAL NORMAL MODE METHODOLOGY}

The non-linear normal mode formulation is developed for the one-dimensional vibration of one-dimensional continuous systems. It extends previous work (Shaw and Pierre, 1994) in two ways. First, the method developed here is able to handle non-linear inertia terms in a consistent manner. Second, one of the two examples treated in this work deals with "intrinsic" non-linearities which arise from finite deformations, whereas all previous research has dealt with "external" sources of non-linearity, such as attached nonlinear elements.

Consider the vibration of a one-dimensional structural system which occupies a closed subset $\Omega$ of $\mathbb{R}$ (e.g., a beam or a string). Material points in this object are labeled by the independent spatial variable $s$, typically related to the undeformed configuration of the system or to an arc-length coordinate fixed to the neutral axis of an inextensibe beam, and $t$ is taken to be the time variable. Let $u(s, t)$ and $v(s, t)$ denote the displacement and velocity of the material point $s \in \Omega$ at time $t$, respectively. The free vibration of this system can be described by the following first-order equations of motion

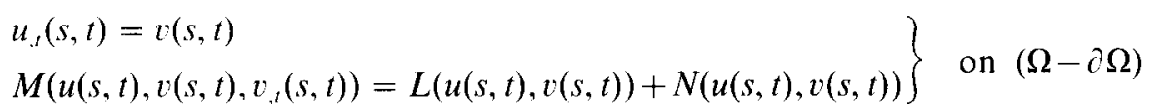

with the following boundary conditions

$$
B(u(s, t), v(s, t))=0 \quad \text { on } \partial \Omega,
$$

where $\partial \Omega$ represents the boundary of the domain $\Omega$, and a subscript following a comma denotes a partial derivative with respect to the corresponding variable. Since the dependence of the functions $u$ and $v$ on the independent variables $s$ and $t$ is often clear, $u(s, t)$ and $v(s, t)$ will be replaced by $u$ and $v$ when no confusion can arise. Here $M\left(u, v, v_{t}\right)$ is an inertia operator, possibly non-linear, which operates linearly on the acceleration $v_{, t}$ and is of arbitrary order in the space variable, $L(u, v)$ and $N(u, v)$ are linear and non-linear spatial stiffness operators, respectively, and $B(u, v)$ denotes the (vector) spatial boundary condition 
operator on $\partial \Omega$. Without loss of generality, the equilibrium state of the system is assumed to be $(u, v)=(0,0)$.

Before proceeding to define the normal modes of motion for such a non-linear system, consider the linearized system associated with eqn (1), whose dynamics are governed by

$$
\left.\begin{array}{l}
u_{, t}(s, t)=v(s, t) \\
M_{l}\left(v_{, t}(s, t)\right)=L(u(s, t), v(s, t))
\end{array}\right\} \quad \text { on }(\Omega-\partial \Omega)
$$

where $M_{l}\left(v_{, t}(s, t)\right)$ denotes the linearized version of the inertial operator $M\left(u, v, v_{, l}\right)$. The boundary condition (2) becomes

$$
B_{l}(u, v)=0 \quad \text { on } \partial \Omega .
$$

Such a linear system typically possess a set of special solutions called natural modes, in which the dynamics of the entire system can be represented by a lower-order, linear system. In vibration applications, these single-mode dynamical systems are governed by ordinary differential equations of second order in time. Each such mode has associated with it an eigenvalue, which contains information about the frequency and the decay rate of the modal motion, and an eigenfunction which describes the configuration of the entire system as it undergoes a purely single mode motion. These natural modes have an arbitrary constant associated with them, and when they are normalized in some manner they are referred to as normal modes. These modes have a special invariance property which can be stated as follows : If the system is unforced and is started in a spatial configuration comprised of a single mode, it will stay in that mode for all time, and no other modes will become active. In terms of the system's phase space, such a motion occurs on a two-dimensional subspace which is invariant. For linear systems, these subspaces are two-dimensional planes, and a general motion can be written as a linear combination of the normal modes motions; this is the principle of superposition.

Only some of these ideas can be generalized to non-linear systems, and, in particular, there is no hope of obtaining a superposition principle. However, the concept of a normal mode motion occurring on an invariant two-dimensional subspace can be extended. These subspaces are not generally planar, but curved, and are referred to as the non-linear normal mode manifolds. They contain the information about how the system displacement and velocity fields are distributed in a single-mode motion and, because these subspaces are not flat, the non-linear mode shapes are amplitude dependent. Furthermore, motions on these manifolds are governed by non-linear oscillators.

The definition of a normal mode motion based on invariant manifolds is as follows (Shaw and Pierre, 1992, 1993).

Definition. A normal mode motion for a non-linear autonomous system [eqns (1) and (2)] is a motion which takes place on a two-dimensional invariant manifold in the phase space of the system. This manifold has the following properties: (1) it passes through the equilibrium point $(u, v)=(0,0)$, and $(2)$, it is tangent to the eigenspace of the associated linearized system [eqns (3) and (4)] at $(u, v)=(0,0)$.

The main characteristic of a normal motion is that the response of the entire system can be described, through the amplitude-dependent mode shape, by the response of a single point on the structure, denoted here as $s_{0}$. This can be stated in mathematical terms as follows: In some neighborhood of the equilibrium point, the displacement and velocity fields can be expressed by

$$
\begin{aligned}
& u(s, t)=U\left(u_{0}(t), v_{0}(t), s, s_{0}\right) \\
& v(s, t)=V\left(u_{0}(t), v_{0}(t), s, s_{0}\right),
\end{aligned}
$$

where $u_{0}(t)=u\left(s_{0}, t\right)$ and $v_{0}(t)=v\left(s_{0}, t\right)$ denote, respectively, the displacement and velocity of the point $s_{0} \in \Omega$ as functions of time. In order to be valid, $U$ and $V$ must also satisfy the boundary conditions, i.e., $B(U, V)=0$ on $\partial \Omega$. It should be noted that eqn (5) can bc considered as a constraint condition which relates the structure's displacement and velocity 
fields, $u$ and $v$, to $u_{0}$ and $v_{0}$ when the system is undergoing a normal mode motion. Also, $U$ and $V$ reduce to linear functions of $u_{0}$ and $v_{0}$ when linearized, recapturing the normal modes for the linearized system in a rather unusual form. Thercfore, in general there are as many solutions for $U$ and $V$ as there are linear normal modes for the system. Moreover, based on this formulation, the following identities must hold at $s=s_{0}$

$$
\begin{aligned}
& U\left(u_{0}(t), v_{0}(t), s_{0}, s_{0}\right)=u_{0}(t) \\
& V\left(u_{0}(t), v_{0}(t), s_{0}, s_{0}\right)=v_{0}(t) .
\end{aligned}
$$

Conditions which ensure that these manifolds are invariant for the equation of motion are now derived, and from these a solution strategy for obtaining approximations of $U$ and $V$ is developed. The approach described here is very similar to that used in the construction of center manifolds (Carr, 1981). The main feature of this construction process is to eliminate the time dependency of the problem in order to obtain equations for $U$ and $V$, from which geometric structure of the manifolds in the phase space can be determined.

A two-dimensional manifold must be parameterized by two independent coordinates and it is quite natural to use the variables $u_{0}$ and $v_{0}$. This results in equations describing the invariant manifolds given by $U$ and $V$ in terms of $u_{0}$ and $v_{0}$. Once the manifolds are determined, the dynamics on them are obtained by confining the equations of motion to these manifolds. The resulting modal oscillators are then used to describe the dynamics of the material point $s=s_{0} \in \Omega$, from which the response of the entire system can be determined from $U$ and $V$.

The construction process begins by taking a time derivative of eqn (6), as follows:

$$
\begin{aligned}
& \frac{\partial U}{\partial t}=\left(\frac{\partial U}{\partial u_{0}}\right) u_{0, t}+\left(\frac{\partial U}{\partial v_{0}}\right) v_{0,} \\
& \frac{\partial V}{\partial t}=\left(\frac{\partial V}{\partial u_{0}}\right) u_{0, t}+\left(\frac{\partial V}{\partial v_{0}}\right) v_{0, t}
\end{aligned}
$$

The constraint condition [eqn (5)] is then enforced everywhere in the equation of motion (1) by replacing $u$ by $U, v$ by $V$, and $u_{, t}$ and $v_{, t}$ by eqn (7), including the boundary conditions. This yields

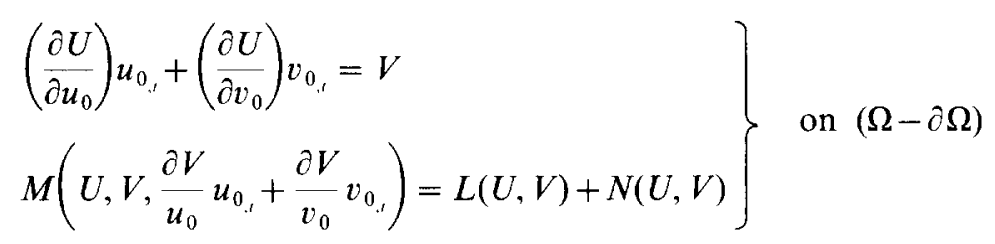

with boundary conditions

$$
B(U, V)=0 \quad \text { on } \partial \Omega
$$

In order to eliminate the time derivatives from these equations, the substitutions $u_{0, t}(t)=v_{0}(t)$ and $v_{0, t}(t)=F_{0}(t)$ are used, where $F_{0}$ is obtained by a procedure described subsequently. The results are

$$
\begin{gathered}
\frac{\partial U}{\partial u_{0}} v_{0}+\frac{\partial U}{\partial v_{0}} F_{0}=V \\
M\left(U, V, \frac{\partial U}{\partial u_{0}} v_{0}+\frac{\partial U}{\partial v_{0}} F_{0}\right)=L(U, V)+N(U, V) .
\end{gathered}
$$

These represent a pair of semi-linear, hyperbolic, partial differential equations. Note that eqns (8) and (9) are valid in a non-local sense, since they are obtained by substituting 
condition (5) into the original equations of motion with the only assumption that the system vibrates in a normal mode motion. The only difficulties at this point are that the functional form for $U$ and $V$ given in eqn (5) may not be valid globally (since the manifold may bend back on itself such that it is not single-valued in terms of $u_{0}$ and $v_{0}$ ) and that these equations are extremely difficult to solve in the general case. However, local solutions can be obtained in the form of asymptotic series in $u_{0}$ and $v_{0}$ if the non-linearities are smooth enough, and the procedure given below allows one to generate these approximate solutions in a systematic manner. If the "global" solution of eqn (10) can be obtained, then the range of validity for these manifolds and their attendant dynamics need not be limited.

The non-linear oscillator is obtained by substituting $(U, V)$ for $(u, v)$ in the equations of motion (1) and evaluating these at $s=a_{0}$. This results in

$$
\begin{aligned}
u_{0, r} & =v_{0} \\
M_{0}\left(u_{0}, v_{0}, v_{0, t}\right) & =\left.M\left(U, V, \frac{\partial V}{u_{0}} v_{0}+\frac{\partial V}{v_{0}} F_{0}\right)\right|_{s=s_{0}}=[L(U, V)+N(U, V)]_{s=s_{0}} .
\end{aligned}
$$

Note that $M_{0}\left(u_{0}, v_{0}, v_{0 .}\right)$ is a linear function of $v_{0}$. In order to determine $F_{0}$, it is required that

$$
\left.M\left(U, V, \frac{\partial V}{u_{0}} v_{0}+\frac{\partial V}{v_{0}} F_{0}\right)\right|_{s=s_{0}}
$$

be invertible with respect to $v_{0, t}$, and this inverse is denoted by $M_{0}^{-1}\left(u_{0}, v_{0}\right)$. In other words, the non-linear modal oscillator is assumed to be expressible in the following form

$$
\begin{aligned}
& u_{0_{. t}}=v_{0} \\
& v_{0_{s}}=M_{0}^{-1}\left(u_{0}, v_{0}\right)[L(U, V)+N(U, V)]_{s=s_{0}}=F_{0}\left(u_{0}, v_{0}\right) .
\end{aligned}
$$

Note that it is not always possible to carry out this inversion explicitly, and in fact, it is usually impossible to obtain $M_{0}^{-1}\left(u_{0}, v_{0}\right)$ in closed form. Nevertheless, here it is assumed that $M_{0}^{-1}\left(u_{0}, v_{0}\right)$ can be determined in some approximated form (e.g., as a series expansion). With this assumption, eqn (12) is achieved.

The local solution procedure for obtaining the normal mode manifolds proceeds by assuming the following asymptotic expansions for $U$ and $V$

$$
\begin{aligned}
U\left(u_{0}, v_{0}, s, s_{0}\right)= & {\left[a_{1}\left(s, s_{0}\right) u_{0}(t)+a_{2}\left(s, s_{0}\right) v_{0}(t)\right] } \\
& +\left[a_{3}\left(s, s_{0}\right) u_{0}^{2}(t)+a_{4}\left(s, s_{0}\right) u_{0}(t) v_{0}(t)+a_{5}\left(s, s_{0}\right) v_{0}^{2}(t)\right]+\cdots \\
V\left(u_{0}, v_{0}, s, s_{0}\right)= & {\left[b_{1}\left(s, s_{0}\right) u_{0}(t)+b_{2}\left(s, s_{0}\right) v_{0}(t)\right] } \\
& +\left[b_{3}\left(s, s_{0}\right) u_{0}^{2}(t)+b_{4}\left(s, s_{0}\right) u_{0}(t) v_{0}(t)+b_{5}\left(s, s_{0}\right) v_{0}^{2}(t)\right]+\cdots
\end{aligned}
$$

which corresponds to a non-linear separation of variables. Since $s_{0}$ is a free parameter, the explicit dependence of $a_{j}$ and $b_{j}$ and $s_{0}$ will be dropped from this point on. Note that the mode shapes, as defined by eqn (13), depend on the magnitudes of $u_{0}$ and $v_{0}$, because the relative contribution of each $a_{j}(s)$ and $b_{j}(s)$ depends on $u_{0}$ and $v_{0}$. This is in contrast with the more typical methods, which separate temporal and spatial behavior at an earlier stage [see, for example, Szemplinska (1990)].

The coefficients $a_{j}(s)$ and $b_{j}(s)$ contain the information for the spatial distribution of the normal mode, while $u_{0}(t)$ and $v_{0}(t)$ govern the time behavior of the normal mode. The solution method for the $a_{j}(s)$ s and $b_{j}(s)$ s proceeds by substituting eqn (13) into eqn (11), subsequently expanding, and gathering the coefficients of like-powered terms in $u_{0}$ and $v_{0}$. This results in a sequence of algebraic-differential equations in the $a_{j}(s) \mathrm{s}$ and $b_{j}(s)$ s. Applying this procedure to the boundary conditions (9) yields a sequence of required boundary conditions for the $a_{j}(s) \mathrm{s}$ and $b_{j}(s)$ s. Together, these equations and boundary conditions 
form a sequence of boundary-value problems which determine the $a_{j}(s)$ and $b_{j}(s)$ values. Moreover, by the nature of this expansion process, these boundary-value problems are uncoupled in sequential order and are linear at all orders except the leading order. As will be shown below, the equations which represent the terms linear in $u_{0}$ and $v_{0}$ recover the linear normal modes, but in an unusual manner. In fact, these are a countable infinity of solutions to the linear order problem, and each acts as a "seed" from which a non-linear normal mode arises.

It is worth noting that the identities given in eqn (7), and the independence of $u_{0}$ and $v_{0}$ require the following conditions to hold for the $a_{j}$ and $b_{j}$ values

$$
\begin{aligned}
& a_{1}\left(s_{0}, s_{0}\right)=1 \\
& a_{j}\left(s_{0}, s_{0}\right)=0, \text { for } j \geqslant 2 \\
& b_{2}\left(s_{0}, s_{0}\right)=1 \\
& b_{j}\left(s_{0}, s_{0}\right)=0, \text { for } j \neq 2 .
\end{aligned}
$$

$\Lambda t$ this point, with a series solution in hand, it is worthwhile to reconsider the inversion of the inertia operator $M$. As previously mentioned, $M_{0}^{-1}$ is typically impossible to obtain in closed form. However, with the assumed series solution for the manifolds [eqn (13)], $M_{0}^{-1}$ can be properly expressed as an asymptotic series in $u_{0}$ and $v_{0}$. This is accomplished by substituting eqn (13) into the inertia operator $M$ and evaluating the result as $s=s_{0}$. This results in

$$
\left.M\left(U, V, \frac{\partial V}{u_{0}} v_{0}+\frac{\partial V}{v_{0}} F_{0}\right)\right|_{s=s_{0}}=\left[m_{0}+m_{1}\left(u_{0}, v_{0}\right)+m_{2}\left(u_{0}, v_{0}\right)+\cdots\right] v_{0, t},
$$

where $m_{j}\left(u_{0}, v_{0}\right)$ is a collection of terms of order $v_{0}^{k} v_{0}^{l}$ with $k+l=j$. Hence, in some local neighborhood of the equilibrium state, $M_{0}^{-1}$ can be written as

$$
M_{0}^{-1}=\left[m_{0}-m_{1}\left(u_{0}, v_{0}\right)-m_{2}\left(u_{0}, v_{0}\right)+\cdots\right] .
$$

Note that, as a consequence, the term $F_{0}$ in eqn (12) can also be expressed in the form of an asymptotic series.

\section{EXAMPLE 1: TRANSVERSE VIBRATION OF A NON-LINEAR CANTILEVER BEAM}

In this section the non-linear normal modes for the free, undamped, transverse vibration of a cantilever beam are constructed. The flexural vibration of the beam is described by a non-linear partial differential equation which contains both geometric and inertia non-linearities up to cubic order.

\subsection{Equation of motion}

The following assumptions are made to derive the equation describing the transverse vibrations of a cantilever beam: (1) the beam is uniform, inextensible and initially straight, (2) plane cross-sections remain plane during deformation, (3) the effects of shear deformation and rotary inertia are neglected, and (4) there exists a longitudinal plane of symmetry and the motion of the beam occurs in this plane. Using these assumptions, the equation of motion is derived using Hamilton's principle.

First, the axial and transverse displacement and velocity fields associated with a general beam motion are considered, from which the potential energy and kinetic energy are obtained. Application of Hamilton's principle yields two partial differential equations in the axial and transverse displacements, which are then reduced to a single differential equation for the transverse motion by making use of an inextensibility constraint (Crespo da Silva and Glynn, 1978). 

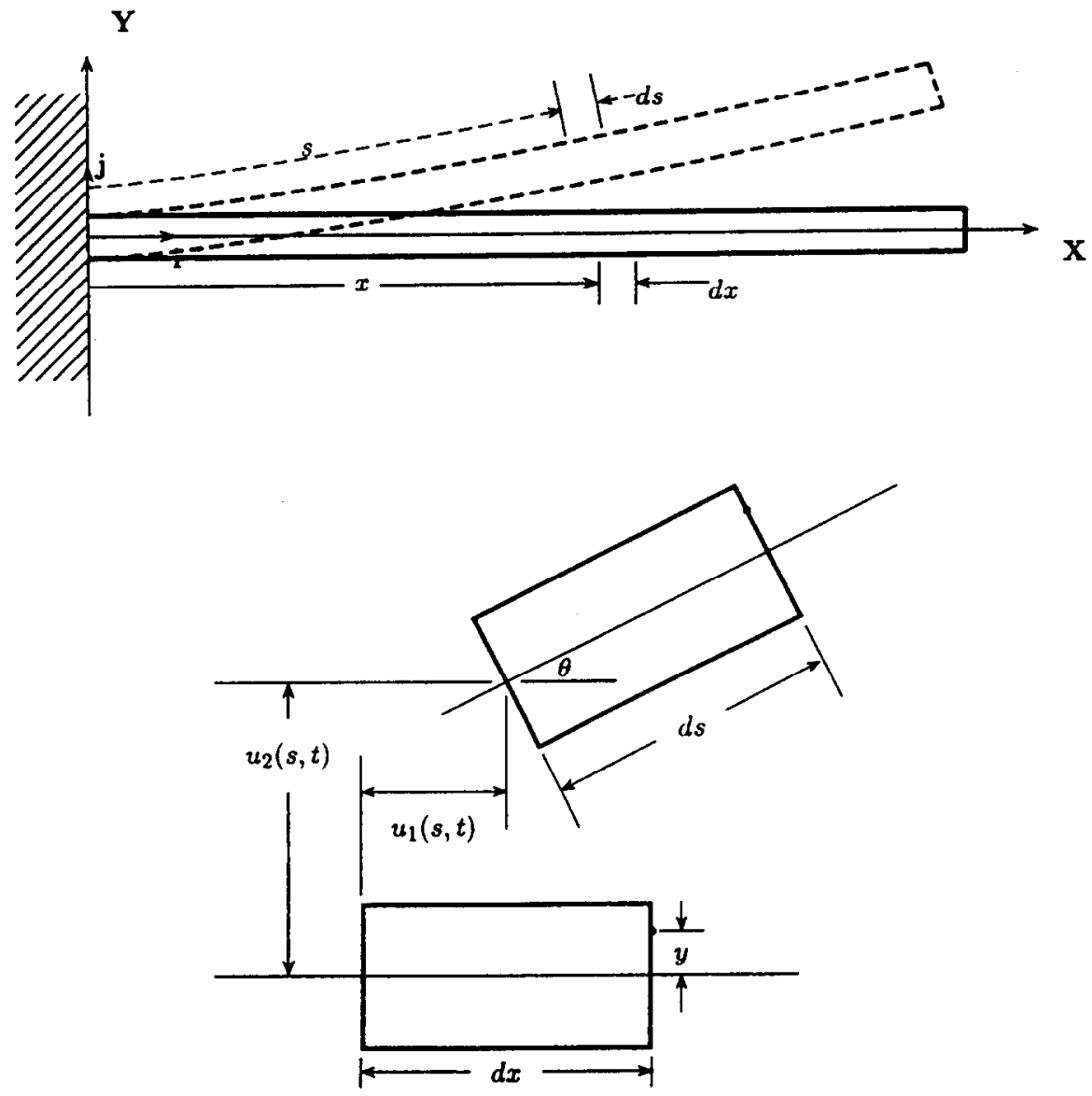

Fig. 1. (a) Cantilever beam; (b) undeformed and deformed beam element.

Consider an infinitesimal beam segment of undeformed length $\mathrm{d} s$ (see Fig. la), where $s$ is the arc-length as measured from the origin of the fixed frame $(O, X, Y)$. After deformation, the (bending) angle between the neutral axis and the $X$-axis is given by $\theta$ (see Fig. $1 b)$. Let a material point located at $(s, y)$ in the undeformed state move to $(\xi, \eta)$. The position vector $\mathbf{r}$ of this point in the deformed state is given as

$$
\begin{aligned}
\mathbf{r}=\xi \mathbf{i}+\eta \mathbf{j} & =\left[s+u_{1}(s, t)-y \sin \theta\right] \mathbf{i}+\left[u_{2}(s, t)+y \cos \theta\right] \mathbf{j} \\
& =\left\{\left[s+u_{1}(s, t)\right] \mathbf{i}+u_{2}(s, t) \mathbf{j}\right\}+[-y \sin \theta \mathbf{i}+y \cos \theta \mathbf{j}],
\end{aligned}
$$

where $\mathbf{i}$ and $\mathbf{j}$ denote the unit vectors along the $O X$ and $O Y$ axes, respectively, and the functions $u_{1}(s, t)$ and $u_{2}(s, t)$ are the displacements of the centroid measured along the $X$ and $Y$ axes, respectively. The Green's strain associated with the material point located at the neutral axis is then given by

$$
\varepsilon_{0}=\left|\frac{\mathrm{d} \mathbf{r}_{y=0}}{\mathrm{~d} s}\right|-1=\left(\sqrt{\left(1+u_{1, s}\right)^{2}+u_{2, s}^{2}}-1\right)
$$

which is obtained by letting $y=0$ in eqn (17) and then taking the derivative with respect to $s$ of the resulting equation. The inextensionality constraint implies no relative elongation of the neutral axis and hence

$$
\left(1+u_{1, s}\right)^{2}+u_{2, s}^{2}-1=0 .
$$


From the geometrical configuration of the deformed element, the following equation for $\theta$ in terms of $u_{1, s}$ and $u_{2, s}$ can be obtained

$$
\tan \theta=\frac{u_{2, s}}{1+u_{1, s}}
$$

Therefore, the Lagrangian associated with the elastic motion of the beam is given as

$$
h=\frac{\rho A}{2} \int_{0}^{l}\left(u_{1 .,}^{2}+u_{2, t}^{2}\right) \mathrm{d} \xi-\frac{E I}{2} \int_{0}^{l} \theta_{. s} \mathrm{~d} s-\frac{\Lambda}{2} \int_{0}^{l}\left[\left(1+u_{1, \xi}\right)^{2}+u_{2, \xi}^{2}-1\right] \mathrm{d} \xi,
$$

where $\rho$ and $E I$ represent, respectively, the mass density and the flexural stiffness of the beam. Note that, in eqn (21), the first term represents the kinetic energy, the second term denotes the potential energy and the last term corresponds to the constraint condition with Lagrange multiplier $\Lambda$ ( $\Lambda$ is the longitudinal force acting along the beam).

In evaluating the Lagrangian, $\theta$ and $\theta_{, s}$ need to be expressed in terms of $u(s, t)$ and $v(s, t)$. The following equations are obtained by expanding and differentiating eqn (20) with respect to $s$ (Crespo da Silva and Glynn, 1978)

$$
\begin{aligned}
\theta & \approx u_{2, s}\left(1+\frac{u_{2, s}^{2}}{6}\right) \\
\theta_{, s} & =u_{2, s s}\left(1+u_{1, s}\right)-u_{2, s} u_{1, s s} .
\end{aligned}
$$

Note that eqn (19) is used in simplifying these two equations.

Applying Hamilton's principle and retaining all non-linear terms up to the cubic order, the following equations for $u_{1}(s, t)$ and $u_{2}(s, t)$ are obtained

$$
\begin{aligned}
\rho A u_{1, t t} & =\left[\Lambda\left(1+u_{1, s}\right)+E I u_{2, s s} u_{2, s}\right]_{, s} \\
\rho A u_{2, t t} & =\left[\Lambda u_{2, s}-E I\left(u_{2, s s s}+u_{2, s} u_{2, s s}^{2}\right)\right]_{, s} \\
\left(u_{1, s}+\frac{u_{2, s}^{2}}{2}\right) & =0
\end{aligned}
$$

subject to the following boundary conditions

$$
\begin{aligned}
u_{1}(0, t) & =0 \\
u_{2}(0, t) & =0 \\
u_{2, s}(0, t) & =0 \\
u_{2, s s}(l, t) & =0 \\
\Lambda\left(1+u_{1, s}(l, t)\right)+E I u_{2, s s s}(l, t) u_{2, s}(l, t) & =0 \\
\Lambda u_{2, s}(l, t)-E I\left(u_{2, s s}(l, t)+u_{2, s}(l, t) u_{2, s s}(l, t)^{2}\right) & =0 .
\end{aligned}
$$

Moreover, from eqn (23c) we have

$$
u_{1}(s, t)=-\frac{1}{2} \int_{0}^{s} u_{2, \xi} \mathrm{d} \xi
$$

which arises essentially from foreshortening effects. Equation (23b) can be integrated once, using boundary conditions (24f) and eqn (25), yielding

$$
\Lambda=-E I u_{2 . s} u_{2, s s s}-\int_{l}^{s} \rho A\left(\int_{0}^{\eta} u_{2, \xi}^{2} \mathrm{~d} \xi\right)_{, u} \mathrm{~d} \eta
$$


Substituting eqns (25) and (26) into eqn (23b), a single equation describing the dynamics of $u_{2}(s, t)$ is obtained

$$
\rho A u_{2, t t}+\frac{\rho A}{2}\left[u_{2, s}\left(\int_{l}^{s} \int_{0}^{\delta} u_{2, \xi}^{2} \mathrm{~d} \xi \mathrm{d} \delta\right)_{, t t}\right]_{, s}+E I\left[u_{2, s s s}+u_{2, s}\left(u_{2, s} u_{2, s s}\right)_{, s}\right]_{, s}=0
$$

Equation (27) describes the free transverse vibration of a slender cantilever beam. It contains the following features: (1) non-linear inertial term $\left[u_{2, s}\left(\int_{l}^{s} \int_{0}^{\delta} u_{2, \xi}^{2} \mathrm{~d} \xi \mathrm{d} \delta\right)_{, t}\right]_{, s}$ and (2) the geometrical non-linearity $E I\left[u_{2, s}\left(u_{2, s} u_{2, s s}\right)_{, s}\right]_{, s}$.

By introducing the non-dimensionalized parameters

$$
t^{*}=t \sqrt{\frac{E I}{\rho A l^{4}}}, \quad u^{*}=\frac{u_{2}}{l}, \quad s^{*}=\frac{s}{l},
$$

eqn (27) can be written in the following non-dimensional form

$$
u_{, t}+\left[u_{, s}\left(\int_{l}^{s} \int_{0}^{\delta} u_{, s}^{2} \mathrm{~d} \xi \mathrm{d} \delta\right)_{, t t}\right]_{, s}+u_{s s s s}+\left[u_{, s}\left(u_{, s} u_{, s s}\right)_{, s}\right]_{, s}=0
$$

where all the asterisks are dropped in order to simplify the notation. With this rescaling and condition ( $24 \mathrm{f})$, the boundary conditions associated with this equation simply become

$$
u(0, t)=u_{, s}(0, t)=u_{, s s}(1, t)=u_{, s s s}(1, t)=0 .
$$

\subsection{Construction of the non-linear normal modes}

The process for constructing the normal modes follows that given in Section 2. The non-linear inertia operator and subsequently $v_{0, \ell}$ [see eqn (12)] are expanded in terms of $u_{0}$ and $v_{0}$ at the outset, so that the equations for the non-linear normal modes can be set up in a convenient form. This leads to expressions which contain some terms expanded in $u_{0}$ and $v_{0}$ and some terms still in $U$ and $V$. This procedure is not inconsistent, since the final result is obtained by expanding all quantities in terms of $u_{0}$ and $v_{0}$.

The construction procedure for the normal modes begins by expressing the equation of motion in first-order form

$$
\begin{aligned}
u_{, t} & =v \\
v_{, t}+\left[u_{s} \int_{1}^{s} \int_{0}^{\delta} v_{, \xi} u_{, \xi} \mathrm{d} \xi \mathrm{d} \delta\right]_{, s} & =-u_{, s s s}-\left[u_{, s}\left(u_{, s} u_{, s s}\right)_{, s}\right]_{, s}-\left[u_{, s} \int_{1}^{s} \int_{0}^{\delta} v_{, \xi}^{2} \mathrm{~d} \xi \mathrm{d} \delta\right]_{, s}
\end{aligned}
$$

The modal oscillators are obtained by substituting the assumed form for a normal mode [eqn (13)] into this first-order system and evaluating the resulting equations at $s=s_{0}$. The result of this calculation is

$$
\begin{aligned}
u_{0, t}= & v_{0} \\
v_{0, t}+\left[\left(U_{, s} \int_{1}^{s} \int_{0}^{\delta} V_{, \xi t} U_{, \xi} \mathrm{d} \xi \mathrm{d} \delta\right)_{, s}\right]_{s=s_{0}}= & -\left[U_{, s s s}+U_{, s s}^{3}+4 U_{, s} U_{, s s} U_{, s s}-U_{, s}^{2} U_{, s s s}\right]_{, s=s_{0}} \\
& -\left[\left(U_{, s} \int_{1}^{s} \int_{0}^{\delta} V_{, \xi}^{2} \mathrm{~d} \xi \mathrm{d} \delta\right)_{, s}\right]_{s=s_{0}}
\end{aligned}
$$


The inertia operator $M_{0}$ is to be expanded as

$$
\begin{aligned}
M_{0}\left(u_{0}, v_{0}, v_{0, t}\right) & =v_{0, t}+\left[\left(U_{. s} \int_{1}^{s} \int_{0}^{\delta} V_{, \xi t} U_{, \xi} \mathrm{d} \xi \mathrm{d} \delta\right)_{, s}\right]_{s=s_{0}} \\
& =\left[m_{0}\left(u_{0}, v_{0}\right)+m_{1}\left(u_{0}, v_{0}\right)+m_{2}\left(u_{0}, v_{0}\right)+\cdots\right] v_{0_{, j}}
\end{aligned}
$$

Note that $m_{0}\left(u_{0}, v_{0}\right)=1$ for the present case. Also, since only cubic-order non-linear terms are present, $m_{1}\left(u_{0}, m v_{0}\right)=0$. Substituting the asymptotic forms for $U$ and $V$ [eqn (13)] into eqn (32) and carrying out the expansion yields the expression of $m_{2}\left(u_{0}, v_{0}\right)$

$$
\begin{aligned}
m_{2}\left(u_{0}, v_{0}\right) & =\left[\left(a_{1}^{\prime} \int_{1}^{s} \int_{0}^{\delta} a_{1}^{\prime} b_{2}^{\prime} \mathrm{d} \xi \mathrm{d} \delta\right)_{s} u_{0}^{2}(t)+\left(a_{2}^{\prime} \int_{1}^{s} \int_{0}^{\delta} a_{1}^{\prime} b_{2}^{\prime} \mathrm{d} \xi \mathrm{d} \delta\right)_{s} v_{0}^{2}(t)\right]_{s=s_{0}} \\
+ & {\left[\left(a_{1}^{\prime} \int_{1}^{s} \int_{0}^{\delta} a_{2}^{\prime} b_{2}^{\prime} \mathrm{d} \xi \mathrm{d} \delta\right)_{s} u_{0}(t) v_{0}(t)+\left(a_{2}^{\prime} \int_{1}^{s} \int_{0}^{\delta} a_{1}^{\prime} b_{2}^{\prime} \mathrm{d} \xi \mathrm{d} \delta\right)_{s} u_{0}(t) v_{0}(t)\right]_{s=s_{0}} }
\end{aligned}
$$

which is quadratic in $u_{0}$ and $v_{0}$. Here the primes denote derivatives with respect to $s$. For this problem $m_{2}$ is sufficient for determining non-linear effects on the mode shapes up to cubic order. Hence, the required approximation of $M_{0}^{-1}$ is

$$
M_{0}^{-1}\left(u_{0}, v_{0}\right)=\left[1-m_{2}\left(u_{0}, v_{0}\right)+\cdots\right]
$$

Using this result, the modal oscillators become

$$
\begin{aligned}
u_{0, t}= & v_{0} \\
v_{0_{, t}}= & -\left[U_{, s s s}+4 U_{, s} U_{, s s} U_{, s s s}+U_{, s s}^{3}+U_{, s}^{2} U_{, s s s}+m_{2} U_{, s s s}\right]_{s=s_{0}} \\
& -\left[\left(U_{, s} \int_{1}^{s} \int_{0}^{\delta} V_{, \xi}^{2} \mathrm{~d} \xi \mathrm{d} \delta\right)_{s}\right]_{s=s_{0}}-v_{0}\left[\left(U_{. s} \int_{1}^{s} \int_{0}^{\delta}\left(\frac{\partial V}{\partial u_{0}}\right) U_{. \xi} \mathrm{d} \xi \mathrm{d} \delta\right)_{s}\right]_{s=s_{0}}+\cdots \\
= & F_{0} .
\end{aligned}
$$

Now that $v_{0, t}$ has been obtained, the equations for the geometry of the normal modes can be set up. This is achieved by using eqn (10) in Section 2, a procedure which eliminates the time derivatives in the equations of motion restricted to the normal mode manifolds. The result is

$$
\begin{gathered}
\left(\frac{\partial U}{\partial u_{0}}\right) v_{0}+\left(\frac{\partial U}{\partial v_{0}}\right) F_{0}=V \\
\left(\frac{\partial V}{\partial u_{0}}\right) v_{0}+\left(\frac{\partial V}{\partial v_{0}}\right) F_{0}+\left[U_{s} \int_{a}^{s} \int_{0}^{\delta}\left(\frac{\partial V}{\partial v_{0}}\right)_{, \xi} U_{, \xi} \mathrm{d} \xi \mathrm{d} \delta\right]_{s} F_{0}=-U_{s s s s}-U_{, s s}^{3}-4 U_{, s} U_{s s} U_{s s s} \\
-U_{. s}^{2} U_{, s s s}-\left[U_{, s} \int_{1}^{s} \int_{0}^{\delta}\left(\frac{\partial V}{\partial u_{0}}\right)_{, \xi} U_{, \xi} \mathrm{d} \xi \mathrm{d} \delta\right]_{, s} v_{0}-\left[U_{s} \int_{1}^{s} \int_{0}^{\delta} V_{, \xi}^{2} \mathrm{~d} \xi \mathrm{d} \delta\right]_{s}
\end{gathered}
$$

A local approximate solution is obtained by substituting the series form for $U$ and $V$, eqn (13), into eqn (36), and expanding in terms of $u_{0}$ and $v_{0}$ and collecting the like-powered terms in $u_{0}$ and $v_{0}$. This results in a sequence of algebraic-differential equations in the $a_{j} \mathrm{~s}$ and $b_{j} \mathbf{s}$, which arc solved below. The boundary conditions associated with these equations are obtained by applying the same procedure to the boundary condition operators. 
This yields

$$
a_{j}(0)=a_{j}^{\prime}(0)=a_{j}^{\prime \prime}(1)=a_{j}^{\prime \prime \prime}(1)=0, \quad j=1,2,3, \ldots
$$

Carrying out the above procedure and gathering the coefficients of the linear-order terms in eqn (35) leads to the following equations in $a_{1}, b_{1}, a_{2}$, and $b_{2}$

$$
\begin{array}{r}
a_{1}^{\prime \prime \prime \prime}\left(s_{0}\right) a_{2}(s)+b_{1}(s)=0 \\
a_{1}(s)-b_{2}(s)-a_{2}(s) a_{2}^{\prime \prime \prime \prime}\left(s_{0}\right)=0 \\
a_{1}^{\prime \prime \prime}(s)-b_{2}(s) a_{1}^{\prime \prime \prime}\left(s_{0}\right)=0 \\
a_{2}^{\prime \prime \prime \prime}(s)+b_{1}(s)-b_{2} a_{2}^{\prime \prime \prime \prime}\left(s_{0}\right)=0 .
\end{array}
$$

Note that eqn (14) was used to simplify the final form of eqn (38).

These equations are non-linear, but not in a usual manner: the non-linear terms are quadratic and always involve the product of a function of $s$ and a function evaluated at $s=s_{0}$. The procedure for obtaining a general solution is not obvious and the following special property of the system is used to determine the solution. It is observed that for a non-gyroscopic, conservative system, the displacement and velocity are not coupled and, therefore, $a_{2}(s)=b_{1}(s)=0$. Hence, eqn (38b) implies that $a_{1}(s)=b_{2}(s)$, and eqn (38c) then reduces to

$$
a_{1}^{\prime \prime \prime \prime}(s)-a_{1}(s) a_{1}^{\prime \prime \prime \prime}\left(s_{0}\right)=0
$$

From the construction procedure, it is very clear that $a_{1}^{\prime \prime \prime \prime}\left(s_{0}\right)$ is a constant coefficient. This makes the eqn (39) equivalent to a linear boundary-value problem for the mode shapes of a linear cantilever beam, in which the eigenvalues apparently depend on the solution evaluated at $s=s_{0}$. This is not the case, however, since a consistent solution can be obtained by simply requiring a particular scaling on the eigenfunctions of the system, as follows.

Denoting the linear mode shapes, i.e., the eigenfunctions, by $\phi_{j}(s)(j=1,2,3, \ldots)$, it can be seen that the solution of $a_{1}(s)$ for eqn (39) is given by

$$
a_{1}(s)=\phi_{j}(s) / \phi_{j}\left(s_{0}\right)
$$

where

$$
\begin{gathered}
\phi_{j}(s)=\left[\cos \lambda_{j} s-\cosh \lambda_{j} s+d\left(\sin \lambda_{j} s-\sinh \lambda_{j} s\right)\right] \\
d=\frac{\cos \lambda_{j}+\cosh \lambda_{j}}{\sin \lambda_{j}+\sinh \lambda_{j}}
\end{gathered}
$$

and $\lambda_{i}$ are the solutions of

$$
\cos \lambda_{j} \cosh \lambda_{j}+1=0
$$

Equation (43) admits a countable infinity of solutions for $\lambda_{j}$, and for each value of $\lambda_{j}$, there is a corresponding solution $a_{1}(s)$. This form of the linear mode shapes forms the basis from which the non-linear normal modes are obtained. Note that eqns (39) and (43) imply $\lambda_{j}=\left[a_{1}^{\prime \prime \prime \prime}\left(s_{0}\right)\right]^{1 / 4}$.

That $s_{0}$ cannot be a nodal point for $\phi_{j}(s)$ is clear from the solution of $a_{1}(s)$. This makes sense because the approach outlined in the previous section breaks down if $s_{0}$ is chosen at a node. Nevertheless, this restriction does not cause any problem, since the choice of $s_{0}$ is free and can be different for different no.mal modes. 
At the quadratic order, six equations are obtained by gathering the coefficients of $u_{0}^{2}$, $u_{0} v_{0}$, and $v_{0}^{2}$ from each of the invariance equations (36). After simplification using the linear-order results and eqn (14), these are given by

$$
\begin{aligned}
b_{3}(s)+\lambda_{n}^{4} a_{4}(s) & =0 \\
2 a_{3}(s)-b_{4}(s)+2 \lambda_{n}^{4} a_{5}(s) & =0 \\
a_{4}(s)-b_{5}(s) & =0 \\
a_{3}^{\prime \prime \prime}(s)-a_{1}(s) a_{3}^{\prime \prime \prime \prime}\left(s_{0}\right)-\lambda_{n}^{4} b_{4}(s) & =0 \\
2 b_{3}(s)-2 \lambda_{n}^{4} b_{5}(s)+a_{4}^{\prime \prime \prime \prime}(s)-a_{1}(s) a_{4}^{\prime \prime \prime \prime}\left(s_{0}\right) & =0 \\
b_{4}(s)+a_{5}^{\prime \prime \prime \prime}(s)-a_{1}(s) a_{5}^{\prime \prime \prime \prime}\left(s_{0}\right) & =0 .
\end{aligned}
$$

These are linear, homogeneous equations of the algebraic-differential type. An obvious solution is the trivial solution, and it is easy to see that the trivial solution is indeed the desired solution, since the non-linear terms must vanish in the absence of the non-linearity. Therefore

$$
a_{f}(s)=b_{f}(s)=0, \text { for } j=3,4,5 \text {. }
$$

This is not unexpected since no quadratic non-linearities are present in the system.

At the cubic order, there are eight equations, obtained by gathering coefficients of $u_{0}^{3}$, $u_{0}^{2} v_{0}, u_{0} v_{0}^{2}$, and $v_{0}^{3}$ from each of the invariance eqns (39). One obtains, after simplifications made using the linear and quadratic orders results and eqn (14)

$$
\begin{aligned}
b_{6}(s)+\lambda_{n}^{4} a_{7}(s) & =0 \\
3 a_{6}(s)-b_{7}(s)-2 \lambda_{n}^{4} a_{8}(s) & =0 \\
2 a_{7}(s)-b_{8}(s)-3 \lambda_{n}^{4} a_{8}(s) & =0 \\
a_{8}(s)-b_{9}(s) & =0 \\
a_{6}^{\prime \prime \prime \prime}(s)-a_{1}(s) a_{6}^{\prime \prime \prime \prime}\left(s_{0}\right)-3 \lambda_{n}^{4} a_{6}(s)+2 \lambda_{n}^{8} a_{8}(s) & =g_{1}\left(a_{1}(s)\right) \\
7 \lambda_{n}^{4} a_{7}(s)-6 \lambda_{n}^{8} a_{9}(s)-a_{7}^{\prime \prime \prime}(s)+a_{1}(s) a_{7}^{\prime \prime \prime \prime}\left(s_{0}\right) & =0 \\
a_{8}^{\prime \prime \prime \prime}(s)-7 \lambda_{n}^{4} a_{8}(s)+6 a_{6}(s)-a_{1}(s) a_{8}^{\prime \prime \prime \prime}\left(s_{0}\right) & =g_{2}\left(a_{1}(s)\right) \\
a_{9}^{\prime \prime \prime \prime}(s)-a_{1}(s) a_{9}^{\prime \prime \prime \prime}\left(s_{0}\right)+2 a_{7}(s)-3 \lambda_{n}^{4} a_{9}(s) & =0,
\end{aligned}
$$

where

$$
\begin{aligned}
g_{1}\left(a_{1}(s)\right)= & \lambda_{n}^{4}\left[a_{1}^{\prime}(s) \int_{1}^{s} \int_{0}^{\delta} a_{1}^{\prime}(\xi)^{2} \mathrm{~d} \xi \mathrm{d} \delta\right]_{s}-\lambda_{n}^{4} a_{1}(s)\left[\left(a_{1}^{\prime}(s) \int_{1}^{s} \int_{0}^{\delta} a_{1}^{\prime}(\xi)^{2} \mathrm{~d} \xi \mathrm{d} \delta\right)\right]_{s=s_{1}} \\
& +a_{1}(s)\left[a_{1}^{\prime \prime}\left(s_{0}\right)\right]^{3}-\left[a_{1}^{\prime \prime}(s)\right]^{3}+4 a_{1}(s) a_{1}^{\prime}\left(s_{0}\right) a_{1}^{\prime \prime}\left(s_{0}\right) a_{1}^{\prime \prime \prime}\left(s_{0}\right) \\
& -4 a_{1}^{\prime}(s) a_{1}^{\prime \prime}(s) a_{1}^{\prime \prime \prime}(s)+a_{1}(s)\left[a_{1}^{\prime}\left(s_{0}\right)\right]^{2} a_{1}^{\prime \prime \prime \prime}\left(s_{0}\right)-\left[a_{1}^{\prime}(s)\right]^{2} a_{1}^{\prime \prime \prime}(s) \\
g_{2}\left(a_{1}(s)\right)= & a_{1}(s)\left[\left(a_{1}^{\prime}(s) \int_{1}^{s} \int_{0}^{\delta} a_{1}^{\prime}(\xi)^{2} \mathrm{~d} \xi \mathrm{d} \delta\right)_{s}\right]_{s=s_{0}}-\left[a_{1}^{\prime}(s) \int_{1}^{s} \int_{0}^{\delta} a_{1}^{\prime}(\xi)^{2} \mathrm{~d} \xi \mathrm{d} \delta\right]
\end{aligned}
$$

Note that the functions $g_{1}\left(a_{1}(s)\right)$ and $g_{2}\left(a_{1}(s)\right)$ must be zero at $s=s_{0}$, according to the solution procedure [eqns (6) and (14)]. The coefficients $a_{7}(s)$ and $a_{9}(s)$ are governed by two homogeneous differential equations which admit the trivial solution. In turn, this implies that $b_{6}(s)$ and $b_{8}(s)$ are also zero. Moreover, $b_{7}(s)$ and $b_{9}(s)$ are related to $a_{6}(s)$ and $a_{8}(s)$ by 


$$
\begin{aligned}
& b_{7}(s)=3 a_{6}(s)-2 \lambda_{n}^{4} a_{8}(s) \\
& b_{9}(s)=a_{8}(s)
\end{aligned}
$$

from eqns (46b) and (46d). Hence solving for the coefficients $a_{6}(s)$ and $a_{8}(s)$ [governed by eqns (46e) and (46g)] is sufficient to define all of the cubic-order terms. To solve this nonhomogeneous boundary-value problem, one approach is to express the particular solutions as linear combinations of the linear eigenfunctions $\phi_{j}(s)$, as follows :

$$
a_{6}(s) \approx \sum_{i=1}^{N} h_{6_{i}} \phi_{i}(s), \quad a_{8}(s) \approx \sum_{i=1}^{N} h_{8_{i}} \phi_{i}(s) .
$$

Substituting the assumed expansions into eqns (46e) and (46g), and projecting the resulting equations on to the $\phi_{j}(s)$ values yields a set of linear equations in the constants $h_{6_{i}}$ and $h_{8_{i}}$. Since in practice only a finite number of terms, $N$, is used to approximate $a_{6}(s)$ and $a_{8}(s)$, a critical issue is the convergence of the approximate solution, eqn (49), as $N$ increased. For the cantilever beam considered here, the solutions for $a_{6}(s)$ and $a_{8}(s)$ obtained following the procedure above turns out to have very poor convergence properties, due to the fact that the non-homogeneous terms $g_{1}$ and $g_{2}$ cannot be approximated with reasonable accuracy (say, within 5\%) for a reasonable number of terms [say, $N=80$ in eqn (49)]. This is caused by the fact that $g_{1}\left(a_{1}(s)\right)$ and $g_{2}\left(a_{1}(s)\right)$ do not satisfy the boundary conditions associated with the $\phi_{j}(s)$ values, as shown clearly in Fig. 2.
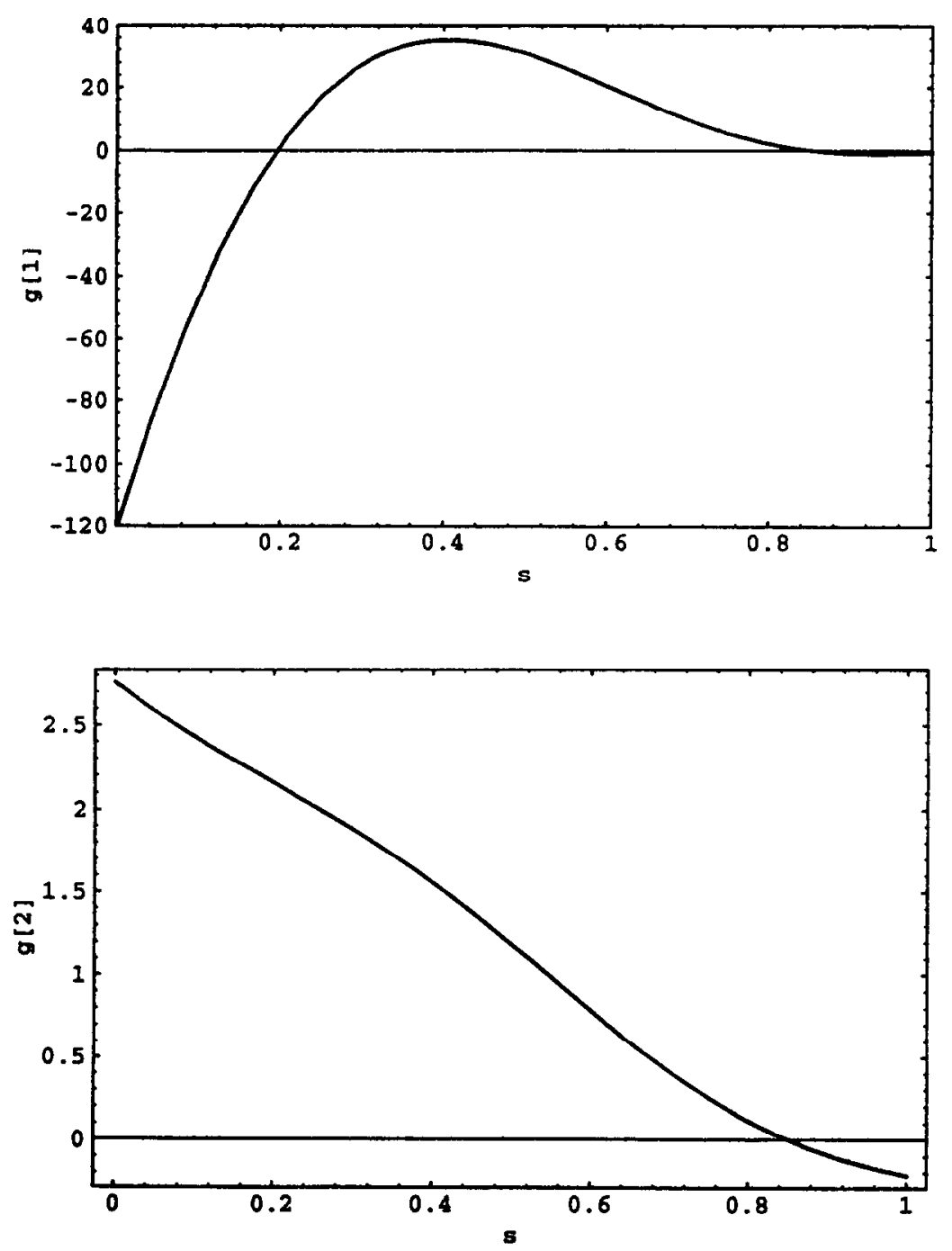

Fig. 2. Plots of functions $g_{1}$ and $g_{2}$, for the first mode with $s_{0}=0.85$. 
In order to achieve improved convergence, $a_{6}(s)$ and $a_{8}(s)$ are solved for in the following alternate way ( $\mathrm{Gu}$ and Tongue, 1987). First, the coefficients $a_{6}$ and $a_{8}$ are expressed as

$$
\begin{aligned}
& a_{6}(s)=\bar{a}_{6}(s)+\eta_{6}(s) \\
& a_{8}(s)=\bar{a}_{8}(s)+\eta_{8}(s),
\end{aligned}
$$

where $\eta_{6}(s)$ and $\eta_{8}(s)$ are simple functions which are chosen to satisfy the non-homogeneous terms at the boundaries. Substituting eqn (50), into eqns (46e) and (46g) leads to

$$
\begin{aligned}
\bar{a}_{6}^{\prime \prime \prime \prime}(s)-3 \lambda_{n} \bar{a}_{6}(s)+2 \lambda_{n}^{8} \bar{a}_{8}(s)-a_{1}(s) \bar{a}_{6}^{\prime \prime \prime \prime}\left(s_{0}\right) & =f_{1}\left(\eta_{6}, \eta_{8}\right) \\
\bar{a}_{8}^{\prime \prime \prime \prime}(s)-7 \lambda_{n}^{4} \bar{a}_{8}(s)+6 \bar{a}_{6}(s)-a_{1}(s) \bar{a}_{8}^{\prime \prime \prime \prime}\left(s_{0}\right) & =f_{2}\left(\eta_{6}, \eta_{8}\right) \\
\eta_{6}^{\prime \prime \prime \prime}(s) & =g_{1}\left(a_{1}(s)\right) \\
\eta_{8}^{\prime \prime \prime \prime}(s) & =g_{2}\left(a_{1}(s)\right),
\end{aligned}
$$

where

$$
\begin{aligned}
& f_{1}\left(\eta_{6}, \eta_{8}\right)=3 \lambda_{n}^{4} \eta_{6}(s)-2 \lambda_{n}^{8} \eta_{8}(s) \\
& f_{2}\left(\eta_{6}, \eta_{8}\right)=7 \lambda_{n}^{4} \eta_{8}(s)-6 \eta_{6}(s) .
\end{aligned}
$$

And the corresponding boundary conditions become

$$
\left.\begin{array}{l}
\bar{a}_{j}(0)=\bar{a}_{j}^{\prime}(0)=\bar{a}_{j}^{\prime \prime}(1)=\bar{a}_{j}^{\prime \prime \prime}(1)=0 \\
\eta_{j}(0)=\eta_{j}^{\prime}(0)=\eta_{j}^{\prime \prime}(1)=\eta_{j}^{\prime \prime \prime}(1)=0
\end{array}\right\} \quad j=6,8 .
$$

Note that the functions $\eta_{6}$ and $\eta_{8}$ satisfy all the boundary conditions associated with $\phi_{j}$ s. This provides a much better modal convergence for approximate series solutions of $\bar{a}_{6}$ and $\bar{a}_{8}$, since the non-homogeneous terms $f_{1}$ and $f_{2}$, which are linear combinations of $\eta_{6}$ and $\eta_{8}$, also satisfy the boundary conditions associated with $\phi_{j}$ s. The solution procedure for $\bar{a}_{6}(s)$ and $\bar{a}_{8}(s)$ begins by solving for $\eta_{6}(s)$ and $\eta_{8}(s)$ with boundary conditions (53). This solution is obtained by integrating eqns (5lc) and (51d) directly four times and choosing the constants of integration so that the above boundary conditions are satisfied. The functions $\eta_{6}(s)$ and $\eta_{8}(s)$ are plotted in Figs 3(a) and 3(b), respectively, for the first linear normal modes based on $s_{0}=0.85$.

Now, the functions $\bar{a}_{6}(s)$ and $\bar{a}_{8}(s)$ are assumed to take the form

$$
\bar{a}_{6}(s)=\sum_{j=1}^{N} k_{6_{i}} \phi_{j}(s), \quad \bar{a}_{8}(s)=\sum_{j=1}^{N} k_{8_{i}} \phi_{j}(s)
$$

Substituting eqn (54) into eqns (51a) and (51b), and projecting the resulting equation on to the $\phi_{j}(s) \mathrm{s}$ lead to linear equations for the coefficients $k_{6}$, and $k_{8_{j}}$. These equations have a different form for the cases $j=n$ and $j \neq n$, due to the product terms involving functions of $s$ and functions of $s_{0}\left[a_{1}(s) \bar{a}_{6}^{\prime \prime \prime \prime}\left(s_{0}\right)\right.$ and $\left.a_{1}(s) \tilde{a}_{8}^{\prime \prime \prime \prime}\left(s_{0}\right)\right]$ in eqns (51a) and (51b). Recall that here $n$ is the non-linear normal mode number and $j$ is the index of the linear eigenfunction component to the $n$th non-linear normal mode. The result is 

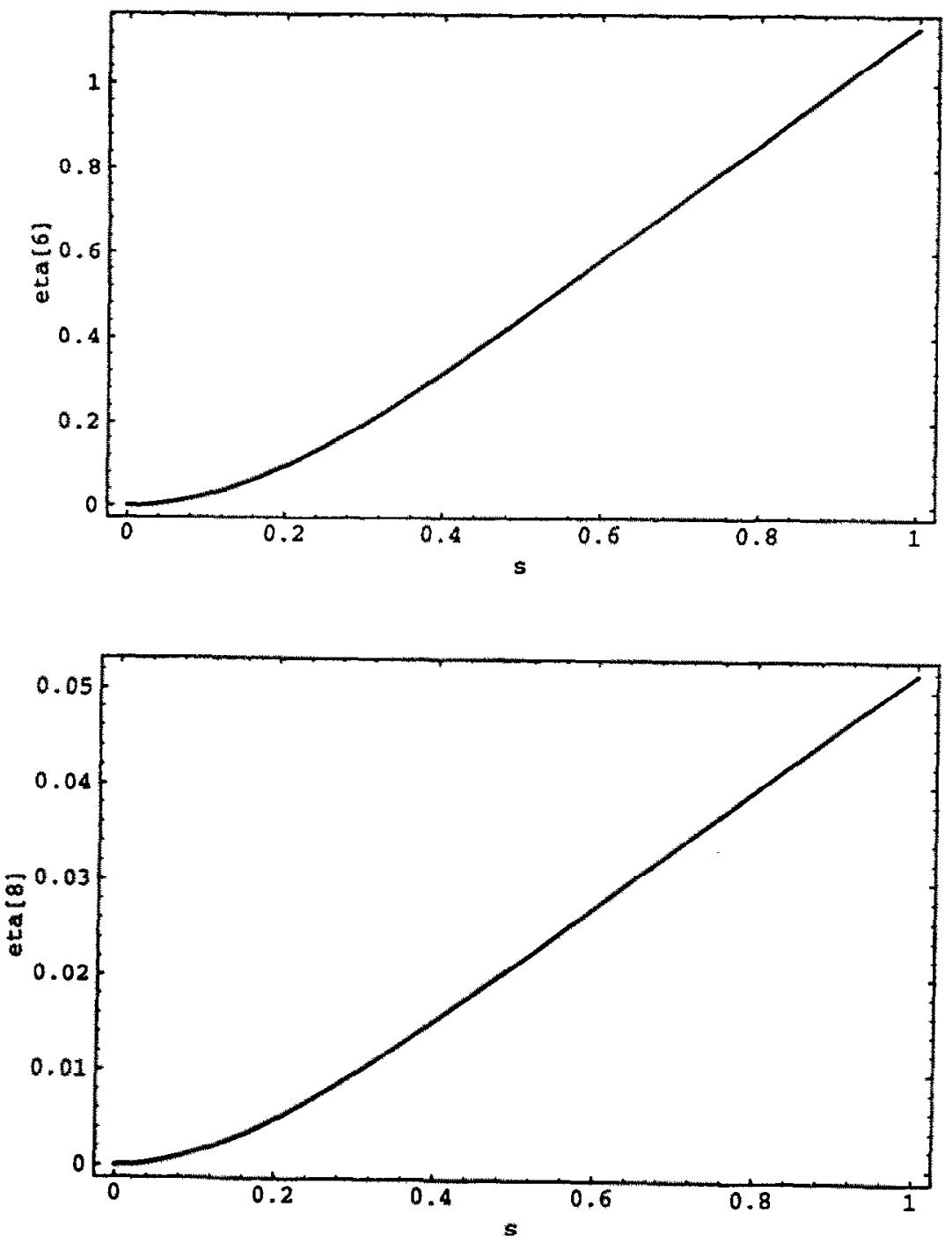

Fig. 3. Plots of solutions $\eta_{5}$ and $\eta_{8}$, for the first mode with $s_{0}=0.85$.

$$
\begin{gathered}
\text { for } j=n\left\{\begin{array}{l}
-2 \lambda_{n}^{4} k_{6_{n}}+2 \lambda_{n}^{8} k_{8_{n}}-\left[\sum_{i=1}^{N} \lambda_{i}^{4} k_{6_{i}} \phi_{i}\left(s_{0}\right) / \phi_{n}\left(s_{0}\right)\right]=f_{t_{n}} \\
6 k_{6_{n}}-6 \lambda_{n}^{4} k_{8_{n}}-\left[\sum_{i=1}^{N} \lambda_{i}^{4} k_{8_{i}} \phi_{i}\left(s_{0}\right) / \phi_{n}\left(s_{0}\right)\right]=\bar{f}_{2_{n}}
\end{array}\right. \\
\text { for } j=n\left\{\begin{array}{l}
\left(\lambda_{j}^{4}-3 \lambda_{n}^{4}\right) k_{6_{j}}+2 \lambda_{n}^{8} k_{8_{i}}=\bar{f}_{\mathrm{i}_{j}} \\
6 k_{6_{j}}+\left(\lambda_{j}^{4}-7 \lambda_{n}^{4}\right) k_{8_{i}}=\bar{f}_{2_{j}}
\end{array}\right\} j=1,2,3, \ldots,
\end{gathered}
$$

where the coefficients $f_{l_{j}}$ and $\vec{f}_{2,}$ are given by

$$
\begin{aligned}
& \tilde{f}_{1_{j}}=\left\langle f_{1}, \phi_{j}(s)\right\rangle=\int_{0}^{1} f_{1}\left(\eta_{6}(s), \eta_{8}(s)\right) \phi_{j}(s) \mathrm{d} s \\
& \tilde{f}_{2,}=\left\langle f_{2}, \phi_{j}(s)\right\rangle \int_{0}^{1} f_{2}\left(\eta_{6}(s), \eta_{8}(s)\right) \phi_{j}(s) \mathrm{d} s
\end{aligned}
$$


in which $\langle\cdot, \cdot\rangle$ represents the usual inner product of square integrable functions over $s=(0,1)$. Equations (55) and (56) form a set of $2 N$ linear equations in $k_{6_{j}}$ and $k_{8}$ for $j=1,2, \ldots, N$. The values of $N$ used to generate the results below are given in Table 1 . Table 2 provides the numerical values for $k_{6_{f}}$ and $k_{8}$ for $j=1,2, \ldots, 8$. Although more than eight terms were used to obtain a satisfactory solution for $\bar{a}_{6}$ and $\bar{a}_{8}$, only the first eight values for $k_{6_{j}}$ and $k_{8,}$ are presented in Table 2 for the sake of brevity.

The use of the alternate procedure described above to obtain $a_{6}(s)$ and $a_{8}(s)$ resulted in a large improvement of the convergence of the series solution. While the direct application of Galerkin's method, eqn (49), necessitated the consideration of at least $N=80$ terms in the series expansion to make the error smaller than $5 \%$, the alternate approach, eqn (52), yields an error smaller than $0.01 \%$ for a 40 -term series. Moreover, it was necessary to use an asymptotic approximation of the higher linear mode shapes (for $j \geqslant 10$ ), as described by Dowell (1984), in order to maintain numerical accuracy for the integrals involved in the calculation of $\bar{f}_{1}$ and $\bar{f}_{2}$.

Due to the complex nature of the functions involved, it was most convenient to obtain solutions in a numerical fashion. The solution procedure is summarized as follows. The linear mode of interest, $\phi_{n}(s)$, is obtained by solving eqn (39). A location for the reference point, $s_{0}$, is then selected for this mode. This defines the solution for $a_{1}$ and hence also the functions $g_{1}$ and $g_{2}$ [eqn (47b)]. The solutions for $\eta_{6}$ and $\eta_{8}$ are then determined by performing the integrals in eqns (51c) and (51d) numerically. This yields the functions $f_{1}$ and $f_{2}$ in eqn (52), which are projected on to the $\phi_{j}(s)$ in order to obtain the coefficients $\bar{f}_{1}$ and $\bar{f}_{2}$, [eqn (57)]. The coefficients $k_{6_{i}}$ and $k_{8_{i}}$ are then calculated by solving the linear equations (55) and (56) for $j=1,2,3, \ldots, N$. Finally, approximate solutions for $a_{6}(s)$ and $a_{8}(s)$ are obtained by combining the solutions for $\eta_{6}(s), \eta_{8}(s), \bar{a}_{6}(s)$, and $\bar{a}_{8}(s)$. Once $a_{6}$ and $a_{8}$ are known, $b_{7}$ and $b_{9}$ are given by eqn (48), and the non-linear mode shapes are then given by eqn (13).

The final form of the approximate nonlinear normal mode shapes are, to cubic order

$$
\begin{aligned}
U_{n}\left(s, s_{0}\right)= & a_{1}(s) u_{0}(t)+\left[\sum_{j=1}^{N} k_{6_{j}} \phi_{j}(s)+\eta_{6}(s)\right] u_{0}(t)^{3} \\
& +\left[\sum_{j=1}^{N} k_{8_{j}} \phi_{j}(s)+\eta_{8}(s)\right] u_{0}(t) v_{0}(t)^{2}+\cdots \\
V_{n}\left(s, s_{0}\right)= & a_{1}(s) v_{0}(t)+b_{7}(s) u_{0}(t)^{2} v_{0}(t)+b_{9}(s) v_{0}^{3}(t)+\cdots
\end{aligned}
$$

where the subscript $n$ denotes the normal mode number whose linearized counterpart is $\phi_{n}$. The expressions for $b_{7}(s)$ and $b_{9}(s)$ are given in terms of $a_{6}(s)$ and $a_{8}(s)$ in eqn (48a) and are not presented here. Note that in the absence of nonlinearities, the linear mode shapes are obtained from eqn (58). Before presenting the examples of these non-linear normal mode results, an important point regarding the range of validity of the asymptotic series for the normal modes [eqn (13)] is discussed.

Table 1. Identification of cases for example 1

\begin{tabular}{ccc}
\hline Fundamental mode & Location of $s_{0}$ & Number of terms used in $a_{6}$ and $a_{8}(N)$ \\
\hline \multirow{3}{*}{$n=1$} & 0.85 & 13 \\
& 0.60 & 13 \\
$n=2$ & 0.30 & 13 \\
& 0.60 & 21 \\
& 0.50 & 21 \\
$n=3$ & 0.40 & 21 \\
& 0.70 & 40 \\
& 0.30 & 40 \\
\hline
\end{tabular}


Table 2. Solutions of cases for example 1

\begin{tabular}{|c|c|c|}
\hline$n$ & $s_{\mathbf{0}}$ & Coefficients $k_{6}$, and $k_{8_{j}} \quad(j \leqslant 8)$ \\
\hline 1 & 0.85 & $\begin{array}{c}k_{6_{1}}=-0.5851, \quad k_{8_{1}}=-2.6973 \times 10^{-3}, \quad k_{6_{2}}=1.2462 \times 10^{-3}, \quad k_{8_{2}}=-6.5197 \times 10^{-5}, \\
k_{6_{3}}=-8.0458 \times 10^{-6}, \quad k_{8_{3}}=3.0344 \times 10^{-6}, \quad k_{6_{4}}=-1.9914 \times 10^{-6}, \quad k_{8_{4}}=3.9635 \times 10^{-7}, \\
k_{6_{5}}=-2.5640 \times 10^{-7}, \quad k_{8_{5}}=4.9834 \times 10^{-8}, \quad k_{6_{6}}=-4.9480 \times 10^{-8}, \quad k_{8_{6}}=9.3672 \times 10^{-9}, \\
k_{6_{7}}=-1.1848 \times 10^{-8}, \quad k_{8_{7}}=2.2280 \times 10^{-9}, \quad k_{6_{8}}=-3.4878 \times 10^{-9}, \quad k_{8_{8}}=6.5014 \times 10^{-10}\end{array}$ \\
\hline 1 & 0.60 & $\begin{aligned} k_{6_{1}}=5.8967, \quad k_{8_{1}}=0.1997, \quad k_{6_{2}}=6.3563 \times 10^{-3}, \quad k_{8_{2}}=-3.3253 \times 10^{-4}, \\
k_{6_{3}}=-4.1037 \times 10^{-5}, \quad k_{8_{3}}=1.5477 \times 10^{-5}, \quad k_{6_{4}}=-1.0157 \times 10^{-5}, \quad k_{8_{4}}=2.0216 \times 10^{-6}, \\
k_{6_{5}}=-1.3078 \times 10^{-6}, \quad k_{8_{5}}=2.5418 \times 10^{-7}, \quad k_{6_{6}}=-2.5237 \times 10^{-7}, \quad k_{8_{6}}=4.7777 \times 10^{-8}, \\
k_{6_{7}}=-6.0432 \times 10^{-8}, \quad k_{8_{7}}=1.1364 \times 10^{-8}, \quad k_{6_{8}}=-1.7789 \times 10^{-8}, \quad k_{8_{8}}=3.3160 \times 10^{-9}\end{aligned}$ \\
\hline 1 & 0.30 & $\begin{array}{c}k_{6_{1}}=1452.73, \quad k_{8_{1}}=102.484, \quad k_{6_{2}}=0.2452, \quad k_{8_{2}}=-1.28258 \times 10^{-2}, \\
k_{6_{3}}=-1.5828 \times 10^{-3}, \quad k_{8_{3}}=5.9694 \times 10^{-4}, \quad k_{6_{4}}=-3.9175 \times 10^{-4}, \quad k_{8_{4}}=7.7971 \times 10^{-5}, \\
k_{6_{5}}=-5.0440 \times 10^{-5}, \quad k_{8_{5}}=9.8036 \times 10^{-6}, \quad k_{6_{6}}=-9.7337 \times 10^{-6}, \quad k_{8_{6}}=1.8427 \times 10^{-6}, \\
k_{6_{7}}=-2.3308 \times 10^{-6}, \quad k_{8_{7}}=4.3829 \times 10^{-7}, \quad k_{6_{8}}=-6.8613 \times 10^{-7}, \quad k_{8_{8}}=1.2790 \times 10^{-7}\end{array}$ \\
\hline 2 & 0.60 & $\begin{array}{c}k_{6_{1}}=429.907, \quad k_{8_{1}}=-1.0897, \quad k_{6_{2}}=12.2334, \quad k_{8_{2}}=-1.5896 \times 10^{-2}, \\
k_{6_{3}}=2.4975, \quad k_{8_{3}}=-1.4245 \times 10^{-2}, \quad k_{6_{4}}=-0.3085, \quad k_{8_{4}}=1.6122 \times 10^{-3}, \\
k_{6_{\mathrm{g}}}=-8.2356 \times 10^{-3}, \quad k_{8_{5}}=5.4234 \times 10^{-4}, \quad k_{6_{6}}=-1.1234 \times 10^{-3}, \quad k_{8_{6}}=9.4002 \times 10^{-6}, \\
k_{6_{7}}=-5.3518 \times 10^{-4}, \quad k_{8_{7}}=3.0362 \times 10^{-6}, \quad k_{6_{8}}=-1.1644 \times 10^{-4}, \quad k_{8_{8}}=9.3213 \times 10^{-7}\end{array}$ \\
\hline 2 & 0.50 & $\begin{array}{c}k_{6_{1}}=242.263, \quad k_{8_{1}}=-0.6141, \quad k_{6_{2}}=26.5406, \quad k_{8_{2}}=-6.6075 \times 10^{-3}, \\
k_{6_{3}}=1.4074, \quad k_{8_{3}}=-8.0273 \times 10^{-3}, \quad k_{6_{4}}=-0.1738, \quad k_{8_{4}}=9.0852 \times 10^{-4}, \\
k_{6_{5}}=-4.6410 \times 10^{-3}, \quad k_{8_{5}}=3.0562 \times 10^{-4}, \quad k_{6_{6}}=-6.9871 \times 10^{-4}, \quad k_{8_{6}}=5.2972 \times 10^{-6}, \\
k_{6_{7}}=-3.0159 \times 10^{-4}, \quad k_{8_{7}}=1.711 \times 10^{-6}, \quad k_{6_{8}}=-9.2654 \times 10^{-5}, \quad k_{8_{8}}=5.2528 \times 10^{-7}\end{array}$ \\
\hline 2 & 0.40 & $\begin{array}{c}k_{6_{1}}=275.813, \quad k_{8_{1}}=-0.6991, \quad k_{6_{2}}=8.8631, \quad k_{8_{2}}=-2.4175 \times 10^{-3}, \\
k_{6_{3}}=1.6023, \quad k_{8_{3}}=-9.1389 \times 10^{-3}, \quad k_{6_{4}}=-0.1979, \quad k_{8_{4}}=1.0343 \times 10^{-3}, \\
k_{6_{5}}=-5.2837 \times 10^{-3}, \quad k_{8_{5}}=3.4794 \times 10^{-5}, \quad k_{6_{6}}=-7.9548 \times 10^{-4}, \quad k_{8_{6}}=6.0308 \times 10^{-6}, \\
k_{6_{1}}=-3.4335 \times 10^{-4}, \quad k_{8_{7}}=1.9480 \times 10^{-6}, \quad k_{b_{8}}=-1.0549 \times 10^{-4}, \quad k_{8_{8}}=5.9802 \times 10^{-7}\end{array}$ \\
\hline 3 & 0.70 & $\begin{array}{c}k_{6_{1}}=-5230.98, \quad k_{8_{1}}=1.3047, \quad k_{6_{2}}=-56.2353, \quad k_{8_{2}}=2.6766 \times 10^{-2}, \\
k_{6_{3}}=-102.275, \quad k_{8_{3}}=-1.6056 \times 10^{-2}, \quad k_{6_{4}}=8.83156, \quad k_{8_{4}}=-1.1039 \times 10^{-2}, \\
k_{6_{5}}=42.2353, \quad k_{8_{5}}=-3.2464 \times 10^{-3}, \quad k_{6_{6}}=0.8788, \quad k_{8_{6}}=-6.5958 \times 10^{-4} \\
k_{6_{7}}=0.3313, \quad k_{8_{7}}=-2.2028 \times 10^{-4}, \quad k_{6_{8}}=5.2080 \times 10^{-2}, \quad k_{8_{8}}=-3.7543 \times 10^{-5}\end{array}$ \\
\hline 3 & 0.30 & $\begin{array}{c}k_{6_{1}}=3436.74, \quad k_{8_{1}}=-0.8572, \quad k_{6_{2}}=36.9463, \quad k_{8_{2}}=-1.7585 \times 10^{-2}, \\
k_{6_{3}}=101.019, \quad k_{8_{3}}=-1.4312 \times 10^{-2}, \quad k_{6_{4}}=-5.8023, \quad k_{8_{4}}=6.8232 \times 10^{-3}, \\
k_{6_{5}}=-27.7484, \quad k_{8_{5}}=2.1329 \times 10^{-3}, \quad k_{6_{6}}=-0.5774, \quad k_{8_{8}}=4.3334 \times 10^{-4} \\
k_{6_{1}}=-0.2177, \quad k_{8_{1}}=1.4472 \times 10^{-4}, \quad k_{6_{8}}=-3.4216 \times 10^{-2}, \quad k_{8_{8}}=2.4665 \times 10^{-5}\end{array}$ \\
\hline 3 & 0.20 & $\begin{array}{c}k_{6_{1}}=6728.56, \quad k_{8_{1}}=-1.67817, \quad k_{\mathrm{f}_{2}}=72.3348, \quad k_{\mathrm{R}_{2}}=-3.4429 \times 10^{-2} \\
k_{6_{3}}=21.614, \quad k_{8_{3}}=-6.62175 \times 10^{-2}, \quad k_{6_{4}}=-11.3599, \quad k_{8_{4}}=1.3359 \times 10^{-2} \\
k_{6_{5}}=-54.3268, \quad k_{8_{5}}=4.1758 \times 10^{-2}, \quad k_{6_{6}}=-1.1304, \quad k_{8_{6}}=8.4841 \times 10^{-4} \\
k_{6_{7}}=-0.4262, \quad k_{8_{7}}=2.8334 \times 10^{-4}, \quad k_{6_{8}}=-6.6990 \times 10^{-2}, \quad k_{8_{8}}=4.8291 \times 10^{-5}\end{array}$ \\
\hline
\end{tabular}

Results obtained showed that different locations of the reference point $s_{0}$ may result in different shapes for the non-linear normal modes. In principle, there is a continuum of valid values of $s_{0}$ (except at the nodes of $\phi_{n}$ ) and the mode shapes obtained using different $s_{0}$ values should be identical. However, due to the fact that the solutions for the normal modes are sought in the form of truncated asymptotic series, they are not identical for various $s_{0}$, but should match closely over some amplitude range. This necessitates the development of a convergence test for the non-linear mode shapes, which is described in Section 5 .

Normal mode shapes are most easily depicted by representing them at peak amplitudes. This is possible since the normal mode motions are standing waves, a fact easily seen by observing that when $u_{0}$ is zero, $U_{n}$ is zero for all $s$, and when $v_{0}$ is zero, $V_{n}$ is zero for all $s$. 
This implies that, in a non-linear normal mode motion, as in a linear normal mode motion, all points on the beam reach their peaks simultaneously and have zero deflection simultaneously. (This is not true for non-conservative or gyroscopic systems, in which normal modes are generally traveling waves.) Hence, a mode shape is presented by plotting the displacement field $U_{n}$ when $v_{0}=0$, corresponding to a beam configuration with zero velocity and an amplitude of $u_{0}$ at $s=s_{0}$. Note that for $v_{0}=0$ the distortion of the nonlinear mode shape is contained in the $a_{6}(s)$ term, which is made up of contributions from all the lincar modes. The non-linear mode shape is dependent on the amplitude $u_{0}$ in the following way. For small amplitudes, $\left|u_{0}\right| \ll 1, U_{n}$ is dominated by $a_{1}(s) u_{0}(t)$, the linear behavior. As the amplitude $u_{0}$ increases, $a_{6}(s) u_{0}^{3}(t)$ comes into play, gradually changing the mode shape in an amplitude-dependent manner.

The first three linear and non-linear normal mode shapes are compared in Figs 4-6. Figures 4(a), 5(a), and 6(a) show the comparisons between the lowest three non-linear and linear modes, in which foreshortening effects are neglected in the linear modes. Figures 4(b), 5(b), and 6(b) show similar plots, but include the purely kinematic foreshortening effects in both the linear and the non-linear modes. From these figures, the following conclusions
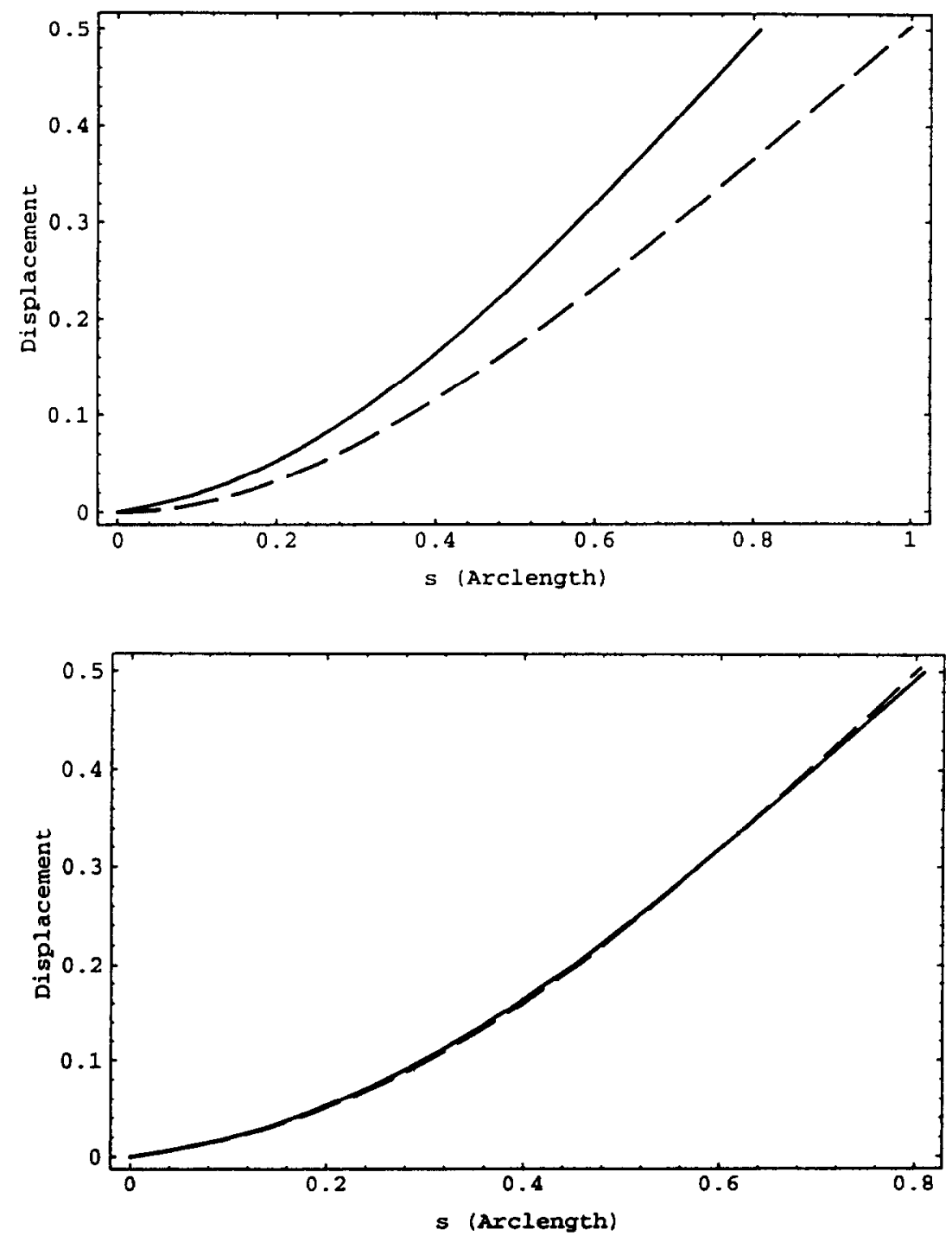

Fig. 4. Comparison of linear and non-linear normal modes (first mode) : (a) linear (dashed line) without foreshortening, non-linear (solid line) with foreshortening; (b) both linear and non-linear with foreshortening. 

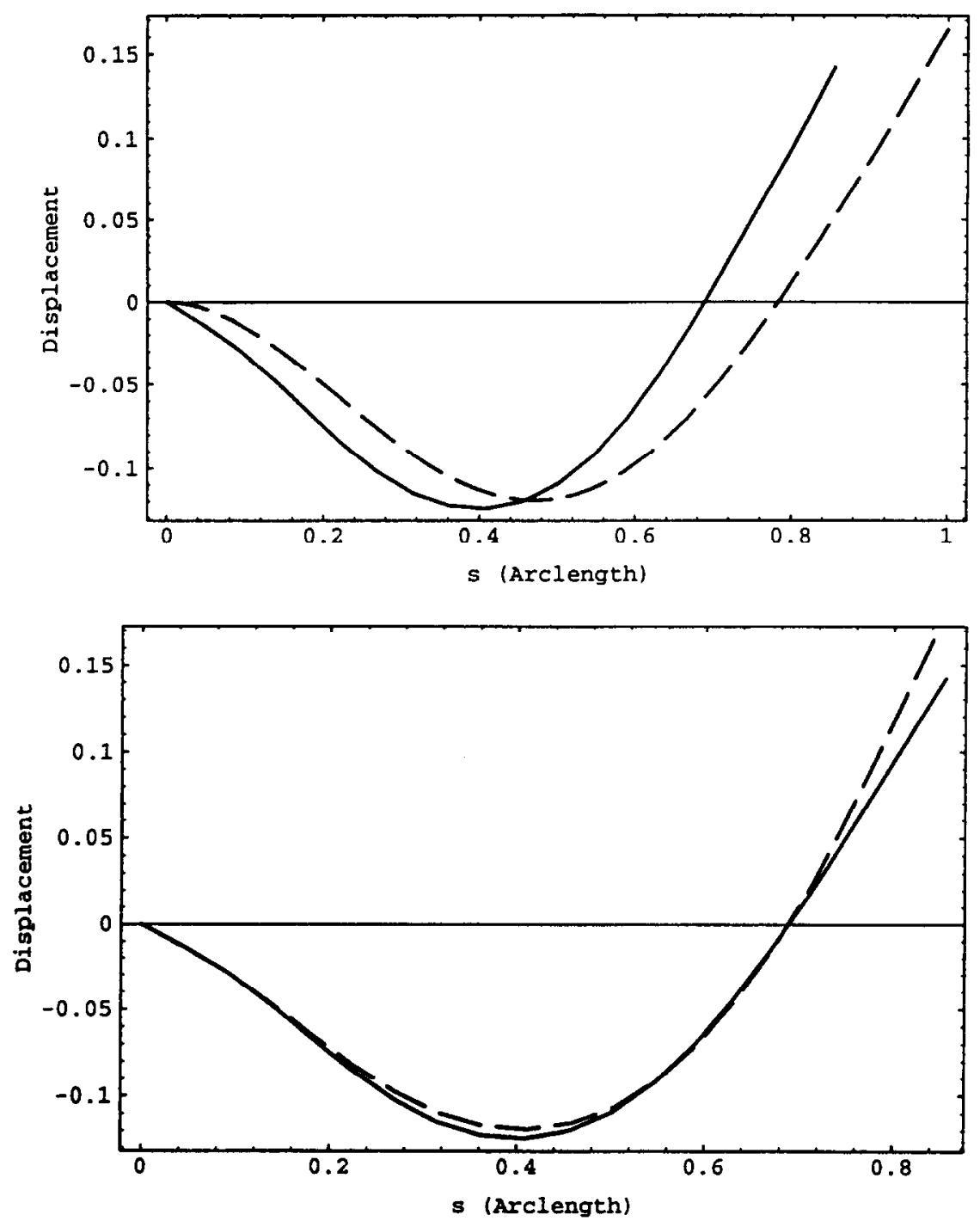

Fig. 5. Comparison of linear and non-linear normal modes (second mode) : (a) linear (dashed line) without foreshortening, non-linear (solid line) with foreshortening; (b) both linear and non-linear with foreshortening.

are drawn. The difference between linear and non-linear modes is significant when the foreshortening effects arc not included. However, the linear and non-linear mode shapes match quite well when the foreshortening effects are included in the linear modes. It is concluded that, for the cantilever beam, the traditional linear normal modes provide an excellent approximation to the maximum beam configuration up to the cubic order by including purely kinematic foreshortening effects.

The construction of these figures is carried out as follows. Each linear and non-linear normal mode is plotted for a given total strain energy at maximum deflection. Note that the transverse displacement is described in terms of the arc-length variable $s$ and the time variable $t$. As a consequence, the resultant non-linear normal mode manifolds are also expressed in terms of the arc-length $s$. Due to foreshortening effects, a conversion between the undeformed state variable, denoted by $x$, and the arc-length $s$ is required for plotting the physical shape of the beam in a normal mode motion. For each normal mode shape, these two variables are related by the following equation

$$
x=\int_{0}^{s} \sqrt{1-\left(U_{. \xi}\right)^{2}} \mathrm{~d} \xi
$$



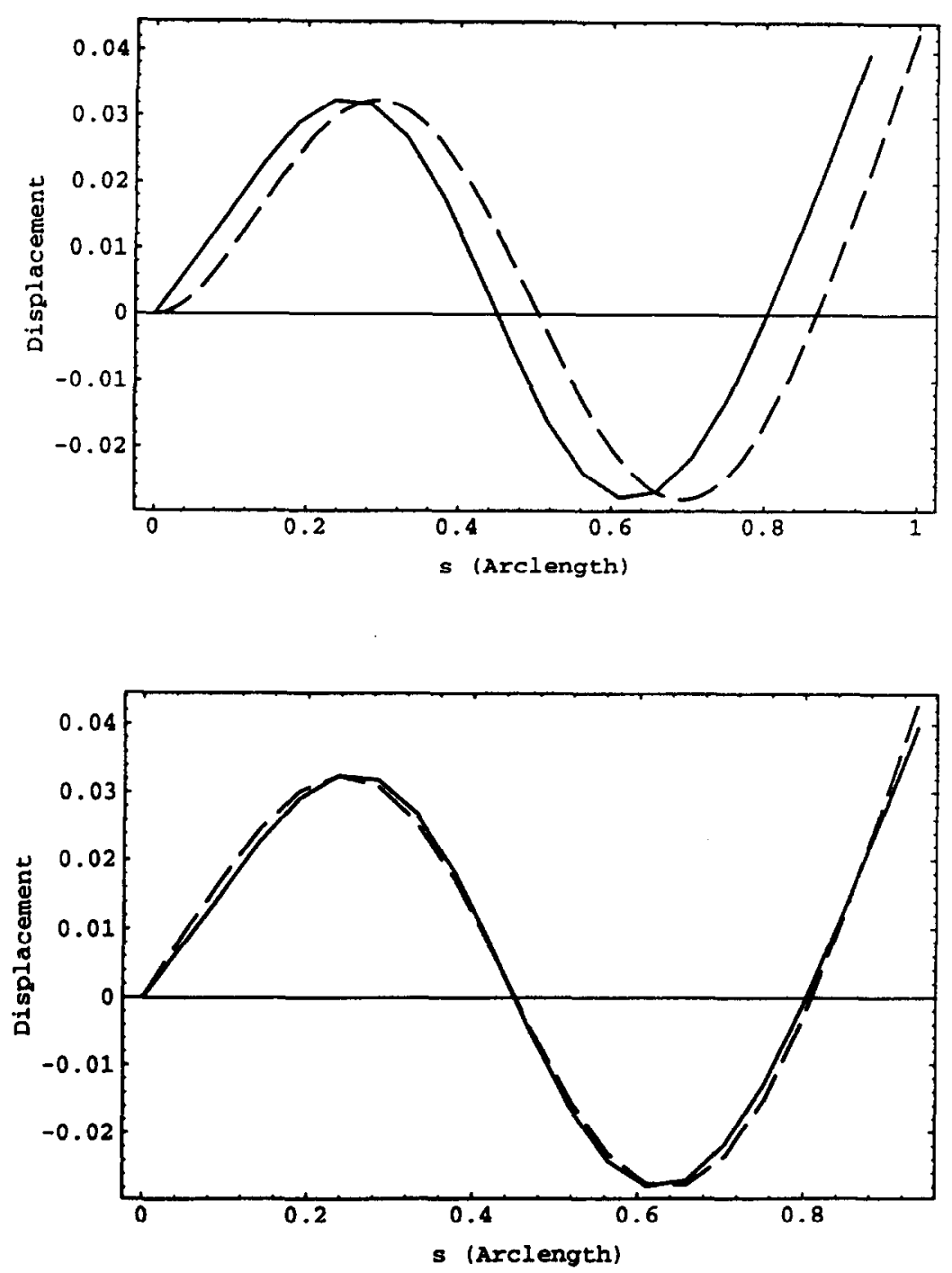

Fig. 6. Comparison of linear and non-linear normal modes (third mode) : (a) linear (dashed line) without foreshortening, non-linear (solid line) with foreshortening; (b) both linear and non-linear with foreshortening.

which yields the identity $x=s$ for the linear mode shapes. In comparing the linear and non-linear mode shapes, non-linear mode shapes are generated by including the effect of foreshortening according to eqn (59). However, in generating the linear mode shapes, two approaches are used. The first approach treats the arc-length $s$ as equal to the undeformed state variable $x$, i.e., foreshortening is ignored. This is valid only when amplitudes are small. In the second approach, the effect of foreshortening is included in generating the linear mode shapes. This is accomplished by simply employing $\phi_{n}$ (with an amplitude set by the strain energy) in place of $u_{n}$ in eqn (59).

The equal strain energy requirement between the linear and non-linear modes is used to relate their amplitudes in the following way

$$
\left|u_{l}\right|^{2} \int_{0}^{1} \phi_{, s s}^{2} \mathrm{~d} s=\int_{0}^{1} U_{, s s}^{2} \mathrm{~d} s
$$

The left- and right-hand sides of eqn (60) represent the strain energy of the linear and the 
non-linear normal modes, respectively. For a given amplitude of $u_{0}(t)$, the corresponding amplitude of the linear mode $u_{l}$ (evaluated at $s_{0}$ ) can be found by solving eqn (60).

The corresponding modal oscillators arc obtained by substituting the expression for $U_{n}$ into eqn (28) and evaluating at $s=s_{0}$. This results in the following non-linear secondorder ordinary differential equations for the first three modes

$$
\begin{aligned}
& n=1, \quad s_{0}=1.00 \\
& \ddot{u}_{0}(t)\left(1+1.60 u_{0}^{2}(t)\right)+12.3624 u_{0}(t)+15.43 u_{0}^{3}(t)+1.21 u_{0}(t) \dot{u}_{0}(t)^{2}+\cdots=0 \\
& n=2, \quad s_{0}=1.00 \\
& \ddot{u}_{0}(t)\left(1+38.75 u_{0}^{2}(t)\right)+485.52 u_{0}(t)-1094.7 u_{0}^{3}(t)+71.36 u_{0}(t) \dot{u}_{0}(t)^{2}+\cdots=0 \\
& n=3, \quad s_{0}=1.00 \\
& \ddot{u}_{0}(t)\left(1+151.67 u_{0}^{2}(t)\right)+3806.5 u_{0}(t)-1.0 \times 1^{6} u_{0}^{3}(t)+819.8 u_{0}(t) \dot{u}_{0}(t)^{2}+\cdots=0 .
\end{aligned}
$$

Here the overdots denote time derivatives. Each of the modal oscillators represents a conservative single degree of freedom system with a $\left(u_{0}, v_{0}\right)$ phase plane comprised of closed orbits. The natural frequencies associated with these modal oscillators are determined by the method of harmonic balance and are given by

$$
\begin{aligned}
& \omega_{1}=3.15602+0.06815\left|u_{0}\right|^{2}+\cdots \\
& \omega_{2}=22.0345-142.22\left|u_{0}\right|^{2}+\cdots \\
& \omega_{3}=61.6972-3453.70\left|u_{0}\right|^{2}+\cdots
\end{aligned}
$$

where $\left|u_{0}\right|$ denotes the displacement at the corresponding reference point $s_{0}$ and $\omega_{n}$ denotes the natural frequency associated with the $n$th non-linear model oscillator. It should be noted that these non-linear frequencies are identical to those obtained by projecting the linear modes on to the non-linear equations of motion and computing the frequencies from the resulting non-linear oscillators [which are different from those given in eqn (63)] (Crespo da Silva and Glynn, 1978). [One must be careful in making these comparisons since the scaling of the amplitude is done quite in an unusual way in the present method, cf. eqn (40).] From these frequencies, it is observed that the non-linear characteristics of the cantilever beam change from hardening to softening at the second mode. This is caused by the competition between the inertial and geometrical non-linearities. For the first mode, the geometrical non-linearity dominates and hence yields hardening cubic non-linearity. For the higher modes, the inertial non-linearity dominates and yields the softening cubic nonlinearity.

\section{EXAMPLE 2: TRANSVERSE VIBRATION OF A LINEAR CANTILEVER BEAM WITH A NON-LINEAR SPRING ATTACHED AT THE FREE END}

The non-linear modes of transverse vibration for a linear cantilever beam with a purely non-linear spring attached at its free end are considered in this section. The purpose of this examplc is to demonstrate the ability of the method to handle problems with discrete nonlinear elements and/or non-linearities in the boundary conditions. 


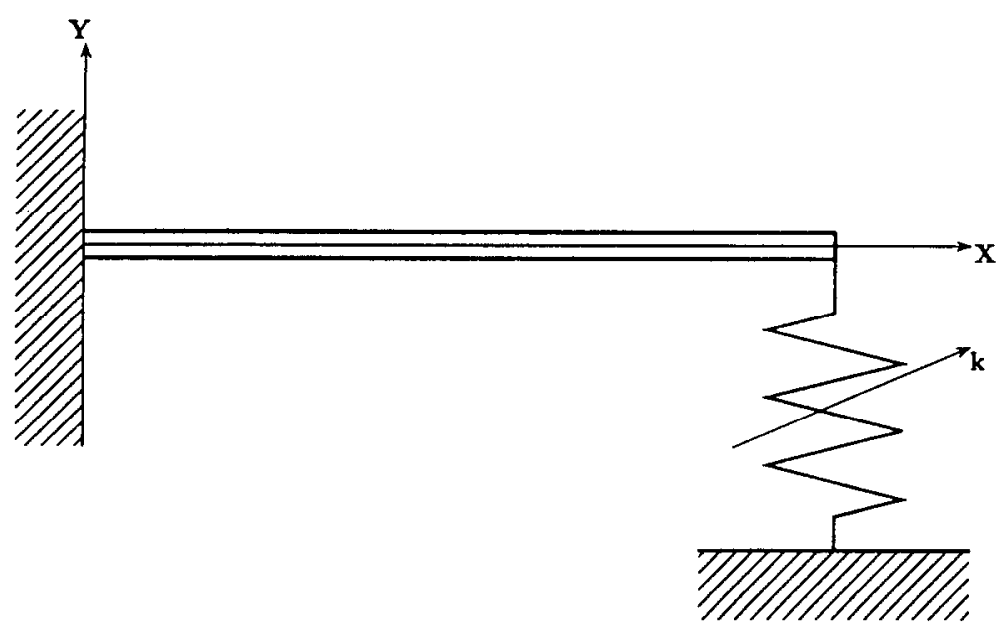

Fig. 7. Cantilever beam with pure non-linear end spring.

The physical system consists of cantilever beam with a non-linear spring attached at its free end (Fig. 7). It is assumed that deflections are small and therefore a linear model is used for the beam. The spring is assumed to be purely non-lin $\mathrm{ar}$, providing a restoring force which is proportional to the cube of the tip displacement, $u(1, t)$. The equation of transverse motion for this system is given by

$$
\rho A u_{, t}+E I u_{, s s s s}=0
$$

with the boundary conditions

$$
u(0, t)=u_{s}(0, t)=u_{. s s}(1, t)=0, \quad E I u_{. s s}=-k u^{3}(1, t),
$$

where $k$ denotes the non-linear spring constant. Introducing the dimensionless parameters

$$
t^{*}=t \sqrt{\frac{E I}{\rho A l^{4}}}, \quad u^{*}=\frac{u}{l}, \quad s^{*}=\frac{s}{l}, \quad \gamma=\frac{k l^{2}}{\rho A},
$$

eqn (65) and the boundary conditions can be rescaled and written in the following dimensionless, first-order form (after dropping all asterisks for notational convenience)

$$
\begin{gathered}
u_{. t}(s, t)=v(s, t), \quad v_{, t}(s, t)=-v_{, s s s}(s, t) \\
u(0, t)=u_{s}(0, t)=u_{s s s}(1, t)=0, \quad u_{s s s}=-\gamma u^{3}(1, t) .
\end{gathered}
$$

The solution procedure begins by substituting the series expansions for the normal mode constraint, eqn (13), into eqn (67), expanding in terms of $u_{0}(t)$ and $v_{0}(t)$, and gathering the coefficients of the like-powered terms in $u_{0}(t)$ and $v_{0}(t)$. This results in a sequence of algebraic-differential equations describing the coefficients $a_{j}$ and $b_{j}$. The boundary conditions associated with these equations are obtained by applying the same procedure to eqn (68).

The linear and quadratic parts of this example are identical to those from the first example, and the same solutions are obtained for coefficients $a_{j}$ and $b_{j}$, for $j=1, \ldots, 5$. Only the results for the cubic order terms are given in detail here. Eight equations arise from the coefficients of the cubic order. After simplifications have been made using the results obtained from the linear and quadratic orders, these eight equations are 


$$
\begin{aligned}
& b_{6}(s)+\lambda_{n}^{4} a_{7}(s)=0 \\
& 3 a_{6}(s)-b_{7}(s)-2 \lambda_{n}^{4} a_{8}(s)=0 \\
& 2 a_{7}(s)-b_{8}(s)-3 \lambda_{n}^{4} a_{9}(s)=0 \\
& a_{8}(s)-b_{9}(s)=0 \\
& a_{6}^{\prime \prime \prime \prime}(s)-a_{6}^{\prime \prime \prime \prime}\left(s_{0}\right) a_{1}(s)-3 \lambda_{n}^{4} a_{6}(s)+2 \lambda_{n}^{8} a_{8}(s)=0 \\
& a_{7}^{\prime \prime \prime \prime}(s)-a_{1}(s) a_{7}^{\prime \prime \prime \prime}\left(s_{0}\right)-7 \lambda_{n}^{4} a_{7}(s)+6 a_{9}(s) \lambda_{n}^{8}=0 \\
& a_{8}^{\prime \prime \prime \prime}(s)-a_{1}(s) a_{8}^{\prime \prime \prime \prime}\left(s_{0}\right)-7 \lambda_{n}^{4} a_{8}(s)+6 a_{6}(s)=0 \\
& a_{9}^{\prime \prime \prime \prime}(s)-a_{1}(s) a_{9}^{\prime \prime \prime \prime}\left(s_{0}\right)-3 \lambda_{n}^{4} a_{9}(s)+2 a_{7}(s)=0,
\end{aligned}
$$

where $\lambda_{n}$ denotes the $n$th eigenvalue associated with the linear cantilever beam and the subscript $n$ indicates the normal mode under consideration. The boundary conditions associated with these equations are

$$
\begin{aligned}
& a_{6}(0)=a_{6}^{\prime}(0)=a_{6}^{\prime \prime}(1)=0, \quad a_{6}^{\prime \prime \prime}(1)=-\gamma a_{1}^{3}(1) \\
& a_{j}(0)=a_{j}^{\prime}(0)=a_{j}^{\prime \prime}(1)=a_{j}^{\prime \prime \prime}(1)=0, \quad \text { for } j=7,8,9 .
\end{aligned}
$$

Note that, by the nature of this system, there are no boundary conditions for coefficient $b_{j}(s)$. The trivial solution for $a_{7}(s)$ and $a_{9}(s)$ satisfies eqns $(69 \mathrm{f})$ and $(69 \mathrm{~h})$ and the associated boundary conditions. Hence, from eqns $(69 \mathrm{a})$ and $(69 \mathrm{c}), b_{6}(s)$ and $b_{8}(s)$ are also zero. Moreover, from eqns (69b) and (69d), $b_{7}(s)$ and $b_{9}(s)$ can be easily solved for in terms of $a_{6}(s)$ and $a_{8}(s)$, as follows:

$$
\begin{aligned}
& b_{7}(s)=3 a_{6}(s)-2 \lambda_{n}^{4} a_{8}(s) \\
& b_{9}(s)=a_{8}(s) .
\end{aligned}
$$

Hence, the coefficients $a_{6}(s)$ and $a_{8}(s)$, as described by eqns $(69 \mathrm{e})$ and $(69 \mathrm{~g})$, are sufficient to define the cubic-order terms of the normal modes.

It is important to note that, in this problem, the governing equations for $a_{6}(s)$ and $a_{8}(s)$ are homogeneous, while their associated boundary conditions are non-homogeneous [equation (70)]. Thus, in order to solve for $a_{6}(s)$ and $a_{8}(s)$, the problem is first transformed into a system of non-homogeneous equations with homogeneous boundary conditions using a standard procedure ( $\mathrm{Gu}$ and Tongue, 1987). Since the non-homogeneity occurs only in the $a_{6}(s)$ boundary conditions, a new function $\bar{a}_{6}(s)$ is defined as follows :

$$
a_{6}(s)=\bar{a}_{6}(s)+\eta_{6}(s),
$$

where $\eta_{6}(s)$ is to be chosen such that the resultant equations in $\bar{a}_{6}(s)$ and $a_{8}(s)$ feature homogeneous boundary conditions. Substituting eqn (72) into eqns $(69 \mathrm{e})$ and $(69 \mathrm{~g})$ and expanding, the following equations are obtained

$$
\begin{aligned}
\bar{a}_{6}^{\prime \prime \prime \prime}(s)-3 \lambda_{j}^{4} \bar{a}_{6}(s)+2 \lambda_{j}^{8} a_{8}(s)-a_{1}(s) a_{6}^{\prime \prime \prime \prime}\left(s_{0}\right) & =3 \lambda_{j}^{4} \eta_{6}(s) \\
a_{8}^{\prime \prime \prime \prime}(s)-7 \lambda_{j}^{4} a_{8}(s)+6 \bar{a}_{6}(s)-a_{1}(s) a_{8}^{\prime \prime \prime \prime}\left(s_{0}\right) & =-6 \eta_{6}(s) \\
\eta_{6}^{\prime \prime \prime \prime}(s) & =0 .
\end{aligned}
$$

Applying this transformation to eqn (70), the following boundary conditions are obtained

$$
\begin{aligned}
& \bar{a}_{6}(0)=\bar{a}_{6}^{\prime}(0)=\bar{a}_{6}^{\prime \prime}(1)=\bar{a}_{6}^{\prime \prime \prime}(1)=0 \\
& a_{8}(0)=a_{8}^{\prime}(0)=a_{8}^{\prime \prime}(1)=a_{8}^{\prime \prime \prime}(1)=0 \\
& \eta_{6}(0)=\eta_{6}^{\prime}(0)=\eta_{6}^{\prime \prime}(1)=0, \quad \eta_{6}^{\prime \prime \prime}(1)=-\gamma a_{1}^{3}(1) .
\end{aligned}
$$


Note that the solution for $\eta_{6}(s)$ is not unique. Here it is chosen to be that corresponding to the transverse deformation of a cantilever beam subjected to a single concentrated end load arising from the non-linear restoring force. In other words, $\eta_{6}(s)$ corresponds to the static solution of a cantilever beam, and is given as

$$
\eta_{6}(s)=\gamma a_{1}^{3}(1)\left(\frac{s^{3}}{6}-\frac{s^{2}}{2}\right)
$$

The functions $\bar{a}_{6}(s)$ and $a_{8}(s)$ are then expressed as a linear combination of the eigenfunctions as follows:

$$
\bar{a}_{6}(s)=\sum_{i=1}^{N} k_{6_{j}} \phi_{j}(s), \quad a_{8}(s)=\sum_{i=1}^{N} k_{8_{j}} \phi_{j}(s)
$$

Substituting eqns (76) into eqns (73a) and (73b) and projecting the resulting equations onto the $\phi_{j}(s) s$, an infinite set of linear algebraic equations in the coefficients $k_{6_{j}}$ and $k_{8_{j}}$ are obtained. Again, due to the nature of the terms involving products of functions of $s$ and $s_{0}$, these equations take different forms for $j=n$ and $j \neq n$. The equations are

$$
\begin{gathered}
k_{6_{j}}\left(\lambda_{j}^{4}-3 \lambda_{n}^{4}\right)+2 \lambda_{n}^{8} k_{8_{i}}=3 \lambda_{j}^{4} \bar{\eta}_{6_{j}}, \quad \text { for } j \neq n \\
-2 \lambda_{n}^{4} k_{6_{n}}+2 \lambda_{n}^{8} k_{8_{n}}-\left(\sum_{i=1}^{N} \lambda_{j}^{4} k_{6_{j}} \phi_{j}\left(s_{0}\right) / \phi_{n}\left(s_{0}\right)\right)=3 \lambda_{n}^{4} \bar{\eta}_{6_{n}}, \quad \text { for } j=n \\
6 k_{6_{i}}+\left(\lambda_{j}^{4}-7 \lambda_{n}^{4}\right) k_{8_{j}}=-6 \bar{\eta}_{6_{j}}, \quad \text { for } j \neq n \\
6 k_{6_{n}}-6 \lambda_{n}^{4} k_{8_{n}}-\left(\sum_{i=1}^{N} k_{8_{j}} \phi_{j}\left(s_{0}\right) / \phi_{n}\left(s_{0}\right)\right)=-6 \bar{\eta}_{6_{n}}, \quad \text { for } j=n,
\end{gathered}
$$

where $\bar{\eta}_{6_{j}}=\left\langle\eta_{6}(s), \phi_{j}(s)\right\rangle$ denotes the component of $\eta_{6}(s)$ projected onto the $j$ th eigenfunction $\phi_{j}(s)$ [eqn (57)]. The coefficients $k_{6_{i}}$ and $k_{8}$, are obtained by solving eqns (77a-d). The solutions for $a_{6}(s)$ and $a_{8}(s)$ are then calculated by combining the solutions of $\eta_{6}(s)$, $\sum k_{6_{i}} \phi_{j}(s)$ and $\sum k_{8}, \phi_{j}(s)$ together in eqn (76). The expressions of $b_{7}(s)$ and $b_{9}(s)$ are given in terms of $a_{6}(s)$ and $a_{8}(s)$ from eqn (71).

Again, due to the complexity of the problem, the solution is obtained numerically. In the current problem, the solution procedure is similar to that outlined in the previous example, except for the presence of the non-linear parameter $\gamma$. The solutions of the nonlinear normal modes are sought for three different $s_{0}$ values (see Table 3), and the numerical values of the first eight coefficients of $k_{6_{i}}$ and $k_{8_{i}}$ are presented in Table 4. The values of $N$ used are given in Table 3.

Table 3. Identification of cases for example 2

\begin{tabular}{ccc}
\hline Fundamental mode & I ocation of $s_{0}$ & Number of terms used in $a_{6}$ and $a_{8}(N)$ \\
\hline \multirow{3}{*}{$n=1$} & 0.95 & 15 \\
& 0.85 & 15 \\
$n=2$ & 0.75 & 15 \\
& 0.50 & 20 \\
& 0.40 & 20 \\
$n=3$ & 0.30 & 20 \\
& 0.70 & 25 \\
& 0.30 & 25 \\
\hline
\end{tabular}


Table 4. Solutions of cases for example 2

\begin{tabular}{|c|c|c|}
\hline$n$ & $s_{0}$ & Coefficients $k_{\delta_{j}}$ and $k_{8_{j}} \quad(j \leqslant 8)$ \\
\hline I & 0.95 & $\begin{array}{c}k_{6_{1}}=0.2055, \quad k_{8_{1}}=-6.9327 \times 10^{-5}, \quad k_{6_{2}}=4.7915 \times 10^{-4}, \quad k_{8_{2}}=-8.3928 \times 10^{-5}, \\
k_{6_{3}}=-6.4884 \times 10^{-6}, \quad k_{8_{3}}=1.0600 \times 10^{-6}, \quad k_{6_{4}}=4.3252 \times 10^{-7}, \quad k_{8_{4}}=-7.0151 \times 10^{-8}, \\
k_{6_{5}}=-5.7703 \times 10^{-8}, \quad k_{8_{5}}=9.3439 \times 10^{-9}, \quad k_{6_{6}}=1.1574 \times 10^{-8}, \quad k_{8_{6}}=-1.8732 \times 10^{-9}, \\
k_{6_{7}}=-3.0399 \times 10^{-9}, \quad k_{8_{7}}=4.91911 \times 10^{-10}, \quad k_{6_{8}}=9.6736 \times 10^{-10}, \quad k_{8_{8}}=-1.5652 \times 10^{-10}\end{array}$ \\
\hline 1 & 0.85 & $\begin{array}{c}k_{6_{1}}=0.3263, \quad k_{8_{1}}=-4.9456 \times 10^{-5}, \quad k_{6_{2}}=7.7353 \times 10^{-4}, \quad k_{8_{2}}=-1.3549 \times 10^{-4}, \\
k_{6_{3}}=-1.0475 \times 10^{-5}, \quad k_{8_{3}}=1.7113 \times 10^{-6}, \quad k_{6_{4}}=6.9825 \times 10^{-7}, \quad k_{8_{4}}=-1.1325 \times 10^{-7}, \\
k_{6_{5}}=-9.3155 \times 10^{-8}, \quad k_{8_{5}}=1.5085 \times 10^{-8}, \quad k_{6_{6}}=1.8685 \times 10^{-8}, \quad k_{8_{6}}=-3.0241 \times 10^{-9}, \\
k_{6_{7}}=-4.9076 \times 10^{-8}, \quad k_{8_{7}}=7.9413 \times 10^{-9}, \quad k_{6_{8}}=1.5617 \times 10^{-9}, \quad k_{8_{8}}=-2.5268 \times 10^{-10}\end{array}$ \\
\hline 1 & 0.75 & $\begin{array}{c}k_{6_{1}}=0.5632, \quad k_{8_{1}}=5.1724 \times 10^{-5}, \quad k_{6_{2}}=1.3595 \times 10^{-3}, \quad k_{8_{2}}=-2.3814 \times 10^{-4}, \\
k_{6_{3}}=-1.8410 \times 10^{-5}, \quad k_{8_{3}}=3.0077 \times 10^{-6}, \quad k_{6_{4}}=1.2272 \times 10^{-6}, \quad k_{8_{4}}=-1.9905 \times 10^{-7}, \\
k_{6_{5}}=-1.6373 \times 10^{-7}, \quad k_{8_{5}}=2.6513 \times 10^{-8}, \quad k_{6_{6}}=3.2839 \times 10^{-8}, \quad k_{8_{6}}=-5.3150 \times 10^{-9}, \\
k_{6_{7}}=-8.6255 \times 10^{-9}, \quad k_{8_{7}}=1.3958 \times 10^{-9}, \quad k_{6_{8}}=2.7448 \times 10^{-9}, \quad k_{8_{8}}=-4.4411 \times 10^{-10}\end{array}$ \\
\hline 2 & 0.50 & $\begin{array}{c}k_{6_{1}}=-0.4541, \quad k_{8_{1}}=-1.6013 \times 10^{-5}, \quad k_{6_{2}}=1.5292 \times 10^{-2}, \quad k_{8_{2}}=7.353 \times 10^{-6}, \\
k_{6_{3}}=-2.6457 \times 10^{-3}, \quad k_{8_{3}}=1.76531 \times 10^{-5}, \quad k_{6_{4}}=-4.9827 \times 10^{-5}, \quad k_{8_{4}}=2.2797 \times 10^{-7}, \\
k_{6_{5}}=5.5013 \times 10^{-6}, \quad k_{8_{5}}=-2.3519 \times 10^{-8}, \quad k_{6_{6}}=-1.0491 \times 10^{-6}, \quad k_{8_{6}}=4.3934 \times 10^{-9}, \\
k_{6_{7}}=2.7038 \times 10^{-7}, \quad k_{8_{7}}=-1.1232 \times 10^{-10}, \quad k_{6_{8}}=-8.5312 \times 10^{-8}, \quad k_{8_{8}}=3.5310 \times 10^{-10}\end{array}$ \\
\hline 2 & 0.40 & $\begin{array}{c}k_{6_{1}}=-0.5170, \quad k_{8_{1}}=-1.8231 \times 10^{-5}, \quad k_{6_{2}}=1.73266 \times 10^{-2}, \quad k_{8_{2}}=-9.2390 \times 10^{-6}, \\
k_{6_{3}}=-3.012 \times 10^{-3}, \quad k_{8_{3}}=2.0098 \times 10^{-5}, \quad k_{6_{4}}=-5.6728 \times 10^{-5}, \quad k_{8_{4}}=2.5954 \times 10^{-7}, \\
k_{6_{5}}=6.2632 \times 10^{-6}, \quad k_{8_{5}}=-2.6776 \times 10^{-8}, \quad k_{6_{6}}=-1.1944 \times 10^{-6}, \quad k_{8_{6}}=5.0018 \times 10^{-9}, \\
k_{6_{7}}=-3.0783 \times 10^{-7}, \quad k_{8_{7}}=-1.2788 \times 10^{-9}, \quad k_{6_{8}}=-9.7127 \times 10^{-8}, \quad k_{8_{8}}=4.0200 \times 10^{-10}\end{array}$ \\
\hline 2 & 0.30 & $\begin{array}{c}k_{6_{1}}=-1.1333, \quad k_{8_{1}}=-3.9964 \times 10^{-5}, \quad k_{6_{2}}=3.9334 \times 10^{-2}, \quad k_{8_{2}}=-5.3441 \times 10^{-5}, \\
k_{6_{3}}=-6.6028 \times 10^{-3}, \quad k_{8_{3}}=4.4057 \times 10^{-5}, \quad k_{6_{4}}=-1.2436 \times 10^{-4}, \quad k_{8_{4}}=5.6895 \times 10^{-7}, \\
k_{6_{5}}=1.3730 \times 10^{-5}, \quad k_{8_{5}}=-5.8698 \times 10^{-8}, \quad k_{6_{6}}=-2.6183 \times 10^{-6}, \quad k_{8_{6}}=1.0965 \times 10^{-8}, \\
k_{6_{7}}=6.7481 \times 10^{-7}, \quad k_{8_{7}}=-2.8032 \times 10^{-9}, \quad k_{6_{8}}=-2.1292 \times 10^{-7}, \quad k_{8_{8}}=8.8124 \times 10^{-10}\end{array}$ \\
\hline 3 & 0.70 & $\begin{array}{c}k_{6_{1}}=-0.5708, \quad k_{8_{1}}=-3.2502 \times 10^{-5}, \quad k_{6_{2}}=1.6139 \times 10^{-2}, \quad k_{8_{2}}=3.7653 \times 10^{-6}, \\
k_{6_{3}}=-2.3224 \times 10^{-3}, \quad k_{8_{3}}=-3.1193 \times 10^{-7}, \quad k_{6_{4}}=8.2808 \times 10^{-5}, \quad k_{8_{4}}=-1.9889 \times 10^{-7}, \\
k_{6_{5}}=2.794 \times 10^{-5}, \quad k_{8_{5}}=-2.0557 \times 10^{-7}, \quad k_{6_{6}}=-1.4966 \times 10^{-5}, \quad k_{8_{6}}=9.019 \times 10^{-9}, \\
k_{6_{7}}=3.1626 \times 10^{-6}, \quad k_{8}=-1.7785 \times 10^{-9}, \quad k_{6_{8}}=-9.2817 \times 10^{-7}, \quad k_{8_{8}}=5.0643 \times 10^{-10}\end{array}$ \\
\hline 3 & 0.30 & $\begin{array}{c}k_{6_{1}}=0.3750, \quad k_{8_{1}}=2.1353 \times 10^{-6}, \quad k_{6_{2}}=-1.1060 \times 10^{2}, \quad k_{8_{2}}=-2.4737 \times 10^{-7} \\
k_{6_{3}}=1.597 \times 10^{-3}, \quad k_{8_{3}}=9.1914 \times 10^{-8}, \quad k_{6_{4}}=-5.440 \times 10^{-5}, \quad k_{8_{4}}=1.3067 \times 10^{-7} \\
k_{6_{5}}=-1.836 \times 10^{-4}, \quad k_{8_{5}}=1.3506 \times 10^{-7}, \quad k_{6_{6}}=9.8329 \times 10^{-6}, \quad k_{8_{6}}=-5.9254 \times 10^{-9} \\
k_{6_{7}}=-2.0778 \times 10^{-6}, \quad k_{8_{7}}=1.1685 \times 10^{-9}, \quad k_{6_{8}}=6.0980 \times 10^{-7}, \quad k_{8_{8}}=-3.3273 \times 10^{-10}\end{array}$ \\
\hline 3 & 0.20 & $\begin{array}{c}k_{6_{1}}=0.7342, \quad k_{8_{1}}=4.1806 \times 10^{-7}, \quad k_{6_{2}}=-2.0761 \times 10^{-2}, \quad k_{8_{2}}=-4.8432 \times 10^{-7} \\
k_{6_{3}}=3.1699 \times 10^{-3}, \quad k_{8_{3}}=-4.0401 \times 10^{-7}, \quad k_{6_{4}}=-1.0652 \times 10^{-4}, \quad k_{8_{4}}=2.5583 \times 10^{-7} \\
k_{6_{5}}=-3.5939 \times 10^{-4}, \quad k_{8_{5}}=2.64423 \times 10^{-7}, \quad k_{6_{6}}=1.9251 \times 10^{-5}, \quad k_{8_{6}}=-1.1601 \times 10^{-8} \\
k_{6_{7}}=-4.0680 \times 10^{-6}, \quad k_{8_{7}}=2.2876 \times 10^{-9}, \quad k_{6_{8}}=1.1939 \times 10^{\circ}, \quad k_{8_{8}}=-6.5142 \times 10^{-10}\end{array}$ \\
\hline
\end{tabular}

The non-linear normal modes are given by, to cubic order

$$
\begin{aligned}
U_{n} & \approx a_{1}(s)+\left[\sum_{i=1}^{N} k_{6_{1}} \phi_{i}(s)+\eta_{6}(s)\right] u_{0}(t)^{3}+\left[\sum_{i=1}^{N} k_{8_{i}} \phi_{i}(s)\right] u_{0}(t) v_{0}(t)^{2}+\cdots \\
V_{n} & \approx a_{1}(s)+b_{7}(s) u_{0}(t)^{2} v_{0}(t)+b_{9}(s) v_{0}^{3}(t)+\cdots
\end{aligned}
$$

where the subscript $n$ denotes the normal mode under consideration.

The first three linear and non-linear modes are compared in Figs 8-10, based again on equal strain energy configurations. The comparison is accomplished by taking a constant 

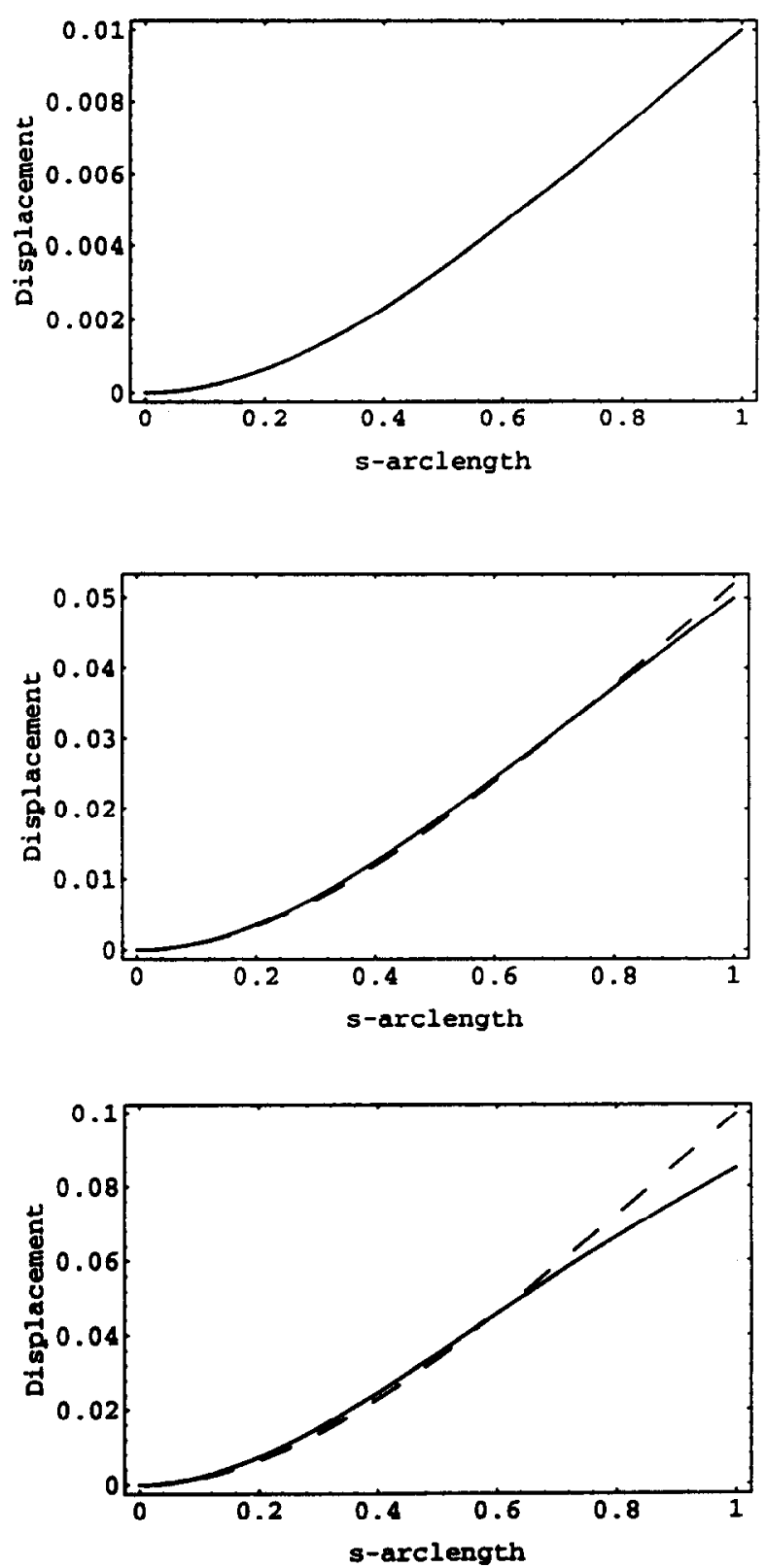

Fig. 8. Comparison of linear and non-linear normal modes (first mode): linear (dashed line), non-linear (solid line).

value of $\gamma$, here 1000 , and then generating the mode shapes for various values of the tip displacement. These figures consist of three linear and non-linear mode shapes each, showing the comparison for tip displacements of $0.01,0.05$, and 0.085 . Note that, in this example, the beam is modeled by linear theory and hence foreshortening effects are not included.

From these figures, it is seen that the non-linear spring has exactly the expected influence on the normal modes. For small amplitudes (Figs 8a, 9a, and 10a) the linear modes are quite accurate, while for large amplitudes (Figs 8c, 9c, and 10c) significant distortions are observed for low order modes.

The corresponding non-linear modal oscillators are obtained by substituting the expressions for $U_{n}$ and $V_{n}$ into the equations of motion, which are then evaluated at $s=s_{0}$. This yields the following non-linear second-order ordinary differential equations for the first three modes 

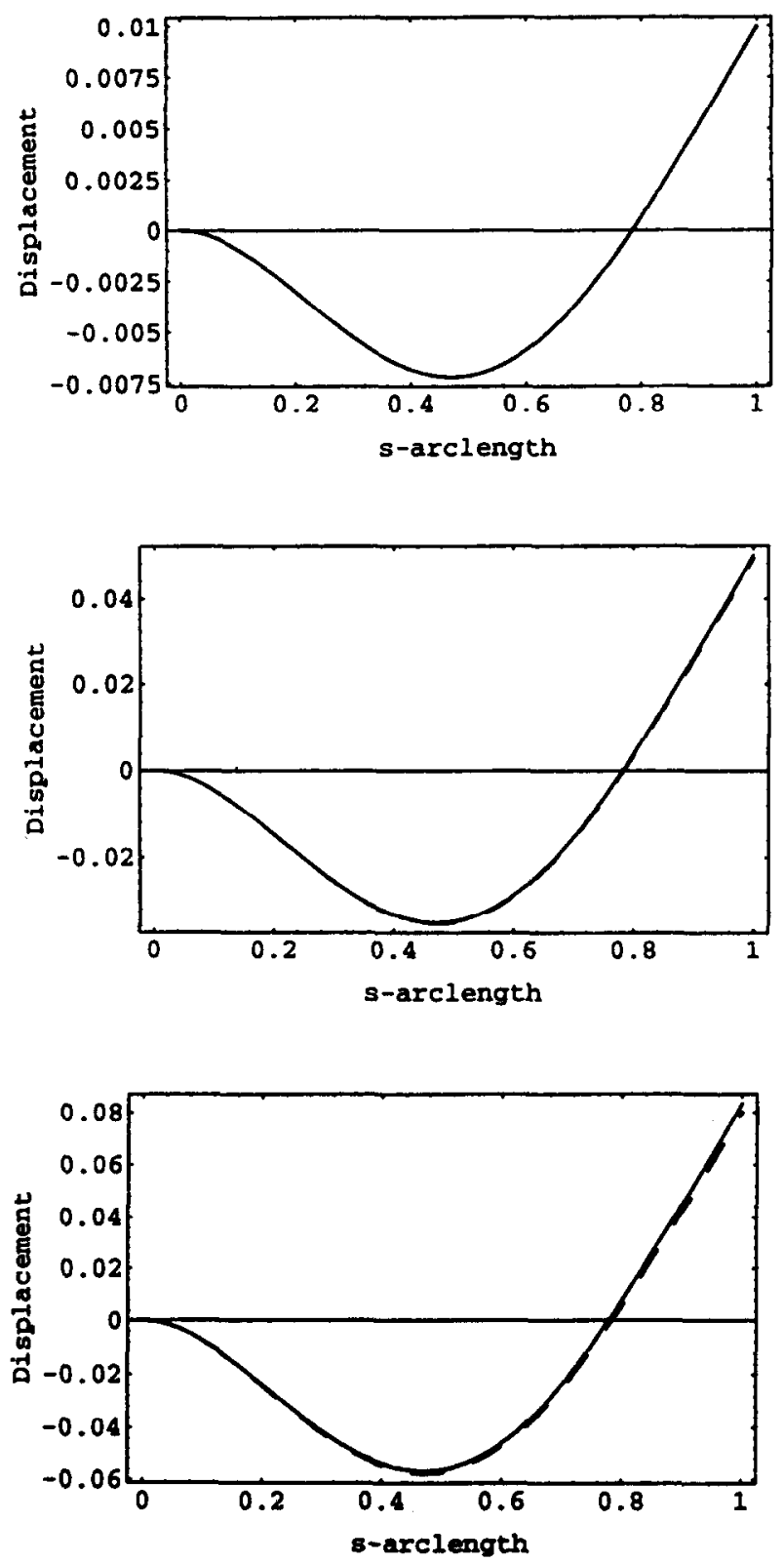

Fig. 9. Comparison of linear and non-linear normal modes (second mode) : linear (dashed line), non-linear (solid line).

$$
\begin{aligned}
& n=1, \quad s_{0}=0.95 \\
& \ddot{u}_{0}(t)+12.3624 u_{0}(t)+3.6977 \gamma u_{0}^{3}(t)+0.073513 \gamma u_{0}(t) \dot{u}_{0}(t)^{2}+\cdots=0 \\
& n=2, \quad s_{0}=0.50 \\
& \ddot{u}_{0}(t)+485.5189 u_{0}(t)+11.1942 \gamma u_{0}^{3}(t)+0.04445 \gamma u_{0}(t) \dot{u}_{0}(t)^{2}+\cdots=0 \\
& n=3, \quad s_{0}=0.70 \\
& \ddot{u}_{0}(t)+3806.55 u_{0}(t)+0.8947 \gamma u_{0}^{3}(t)+0.002447 \gamma u_{0}(t) \dot{u}_{0}(t)^{2}+\cdots=0 .
\end{aligned}
$$

The non-linear frequencies of oscillation associated with these modal oscillators are, to first non-linear order 

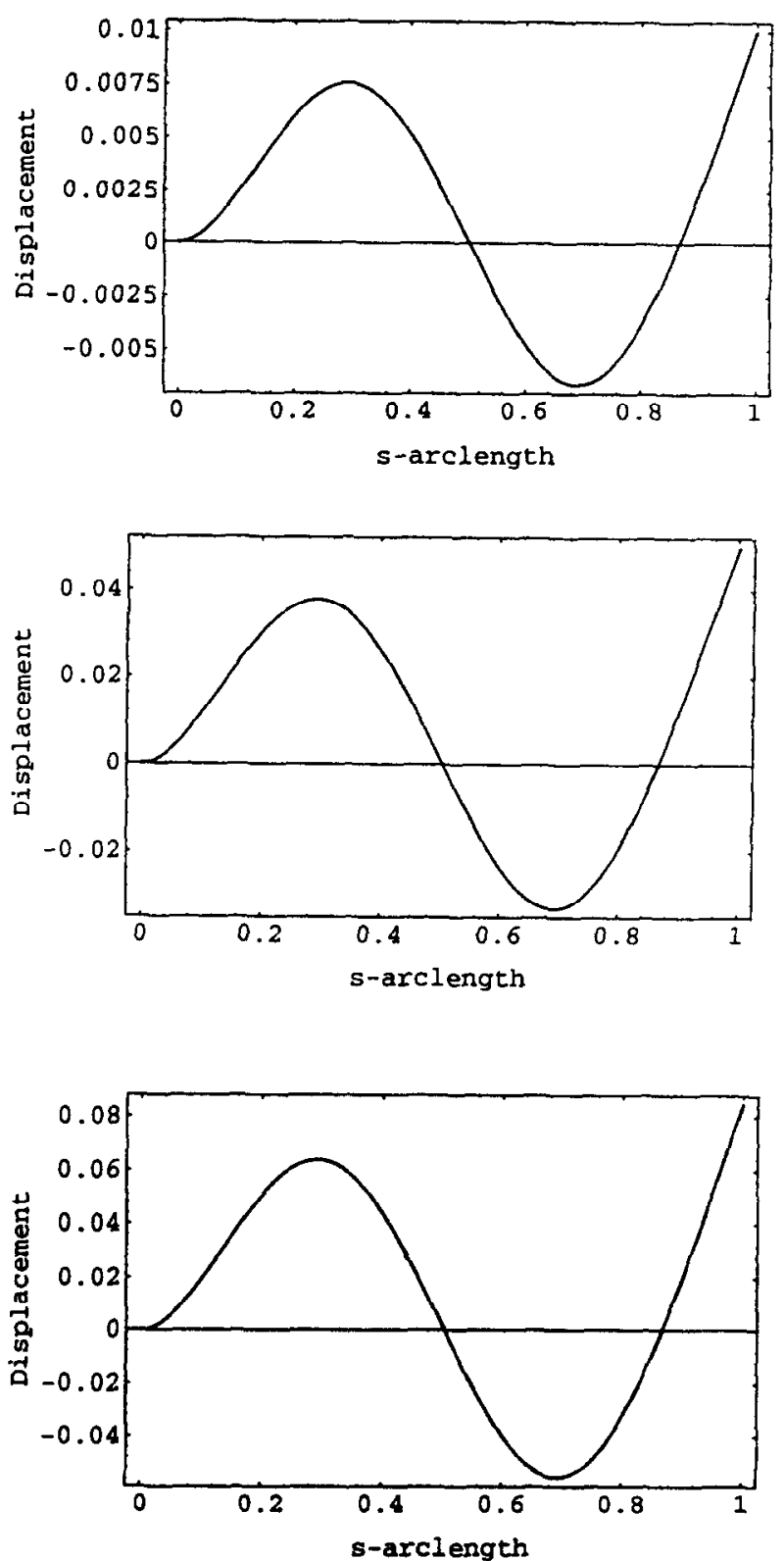

Fig. 10. Comparison of linear and non-linear normal modes (third mode): linear (dashed line), non-linear (solid line).

$$
\begin{aligned}
& \omega_{1}=3.5160+0.4266 \gamma\left|u_{0}\right|^{2}+\cdots \\
& \omega_{2}=22.0345+0.06808 \gamma\left|u_{0}\right|^{2}+\cdots \\
& \omega_{3}=61.6972+0.0243 \gamma\left|u_{0}\right|^{2}+\cdots,
\end{aligned}
$$

where $\omega_{n}$ represents the natural frequency associated with the $n$th non-linear modal oscillator and $\left|u_{0}\right|$ denotes the tip displacement.

\section{CONVERGENCE TESTS FOR THE NON-LINEAR NORMAL MODES}

The dynamic variables $u_{0}$ and $v_{0}$ are the primary parameters which need to be considered with regard to convergence. In the second example, considered in Section 4 , the value of $\gamma$ must also be taken into account, since the magnitude of the non-linearity 
influences the range of convergence. As mentioned in the previous section, normal mode shapes are most easily depicted by showing them at peak amplitudes. Based on this, the convergence of the non-linear normal modes is examined by setting the velocity $v_{0}$ to zero.

A rigorous verification of convergence properties is difficult to perform and therefore it is examined through the following steps :

1. Various reference material points $\left(s_{0}\right)$ are first selected.

2. For each $s_{0}$, the corresponding non-linear normal mode is constructed.

3. These forms for the non-linear normal mode are evaluated based on respective $u_{0}$ values which, if the mode shapes were exact, would render them identical. (The fact that different $u_{0}$ values can lead to a single shape is obvious if one considers a specific beam mode shape and the role of $s_{0}$.)

4. These shapes are plotted together in order to observe the variation amongst them.

Among these four steps, the first two are straightforward, while a few words should be given regarding the last two. Note that the non-linear normal modes are determined based on a particular linear normal mode which has been normalized for different reference points $s_{0}$ [condition (14)]. Based on this observation, the non-linear normal mode shapes are different in scale for different values of $s_{0}$. A normalization is achieved by selecting a non-linear normal mode for a specific $s_{0}$ as the base mode. The other non-linear normal modes, based at different $s_{0}$ points, are then rescaled by proper selection of $u_{0}$ values, so that their respective reference points $s_{0}$ lie on the selected base manifold. In other words, the selected reference points (the $s_{0} \mathrm{~s}$ ) form a sequence of check points along the base nonlinear mode shape. Based on this, the different forms for the non-linear normal mode shapes are plotted on a single graph. Convergence is then examined by observing the splitting between the forms of the mode shapes.

For the first example, $u_{0}$ and $v_{0}$ are the primary parameters required in examining the convergence of the non-linear normal mode shapes. One set of figures is used in examining the convergence between these manifolds, these are generated in the following way. First, the velocity component $v_{0}$ is set to be zero. Then, the value of the tip displacement for the base mode is increased until a considerable difference appears in the plot. Figure 11 shows a typical set of convergent shapes (for the first three modes), and Fig. 12 depicts examples of divergent cases, in which considerable splitting can be observed. This procedure, carried out for each mode with three values of $s_{0}$, is used to determine the upper amplitude limit over which the series solution can be trusted.

For the second example, the figures for convergence test are constructed in the same manner for a fixed value of $\gamma$. Figure 13 shows an example set of convergent shapes for the first three modes, while Fig. 14 shows examples of divergent cases. Note that all the figures shown in Section 4 are based on these convergence tests and the maximum $u_{0}$ values used are chosen to be near the limit values for each mode.

\section{CONCLUSIONS}

The method used in this study was developed originally to treat finite-dimensional problems, based on the concept of invariant manifolds for dynamical systems (Shaw and Pierre, 1992). It was extended to handle infinitc-dimensional, i.e., continuous systems in Shaw and Pierre (1994), but was applied only to systems with "external" non-linear elements, such as the non-linear spring considered in the second example in this work. The present work is the first to employ these methods in the study of "intrinsic" non-linearities which arise purely from large deformation kinematics. In addition, it is shown how the method can be adapted to handlc non-linear inertial operators in the equations of motion. (In the finite-dimensional case, this presents no difficulties, since one simply inverts a matrix using series expansions.)

The conclusions drawn from the specific examples treated here are the following. First, the linear normal modes for cantilever beam represent the actual non-linear normal mode shapes quite accurately over a significant amplitude range (tip displacements up to appproximately $50 \%$ of the beam length) by simply accounting for the kinematic effects of fore- 

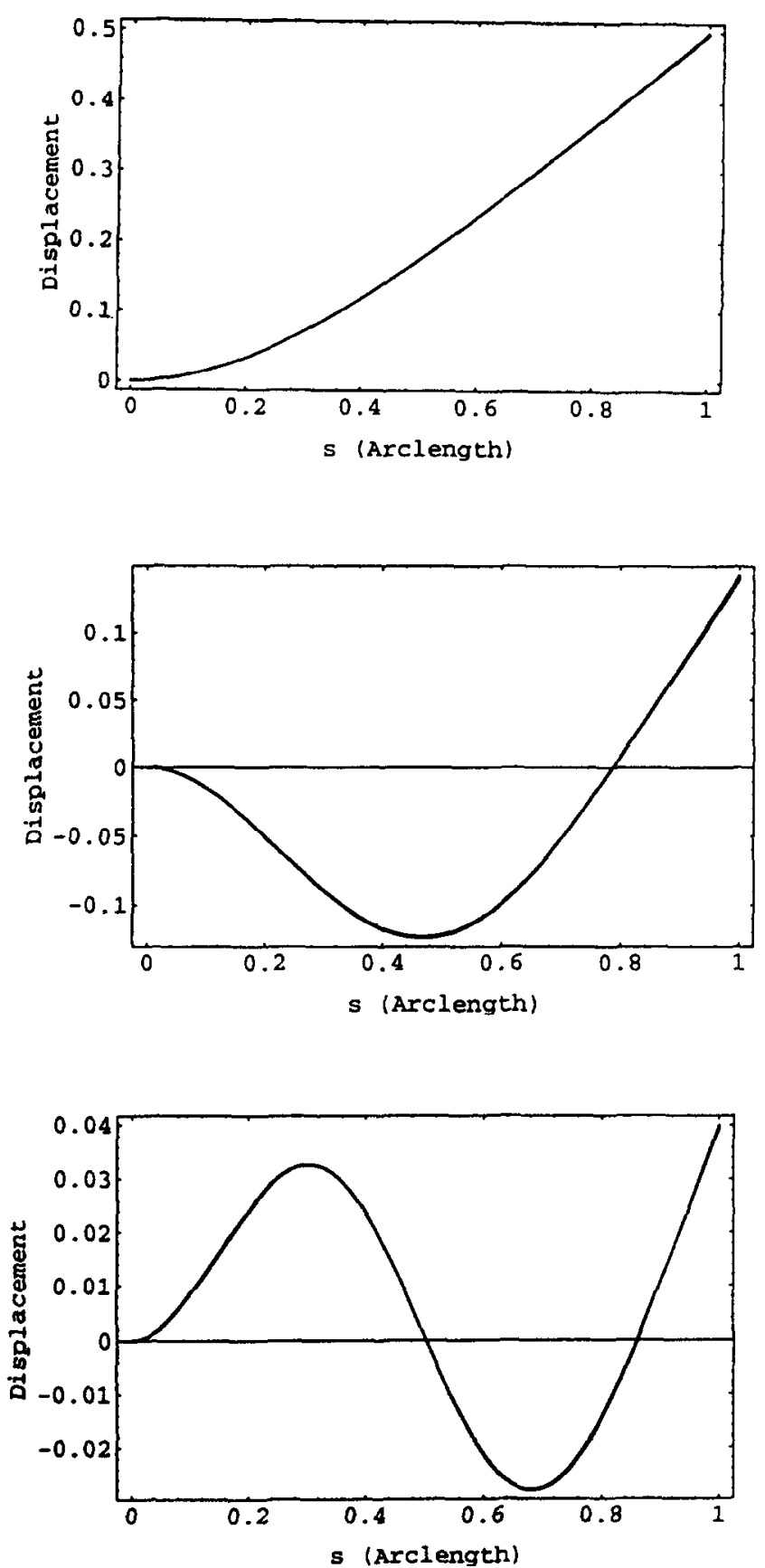

Fig. 11. Typical convergent non-linear normal modes (example 1) : (a) first mode ; (b) second mode ; (c) third mode.

shortening. Thus, it can be concluded that the major source of non-linearity in very flexible beams is kinematic, and not dynamic, in nature. The second example demonstrates that nonlinearities in boundary conditions can have a significant influence on normal mode shapes, and that the present method captures the resulting distortion in a systematic manner.

The two examples presented herein further demonstrate the power and utility of the methodology developed in Shaw and Pierre $(1993,1994)$ in producing non-linear normal modes in a systematic manner which is based on fundamental principles of dynamical systems. It should be noted that the formulation given in Section 2 is quite general, and although only non-gyroscopic, conservative systems which admit standing wave normal modes have been considered, the method is well suited to describing normal modes for nonlinear systems which are non-conservative and/or gyroscopic. The reader is referred to the 

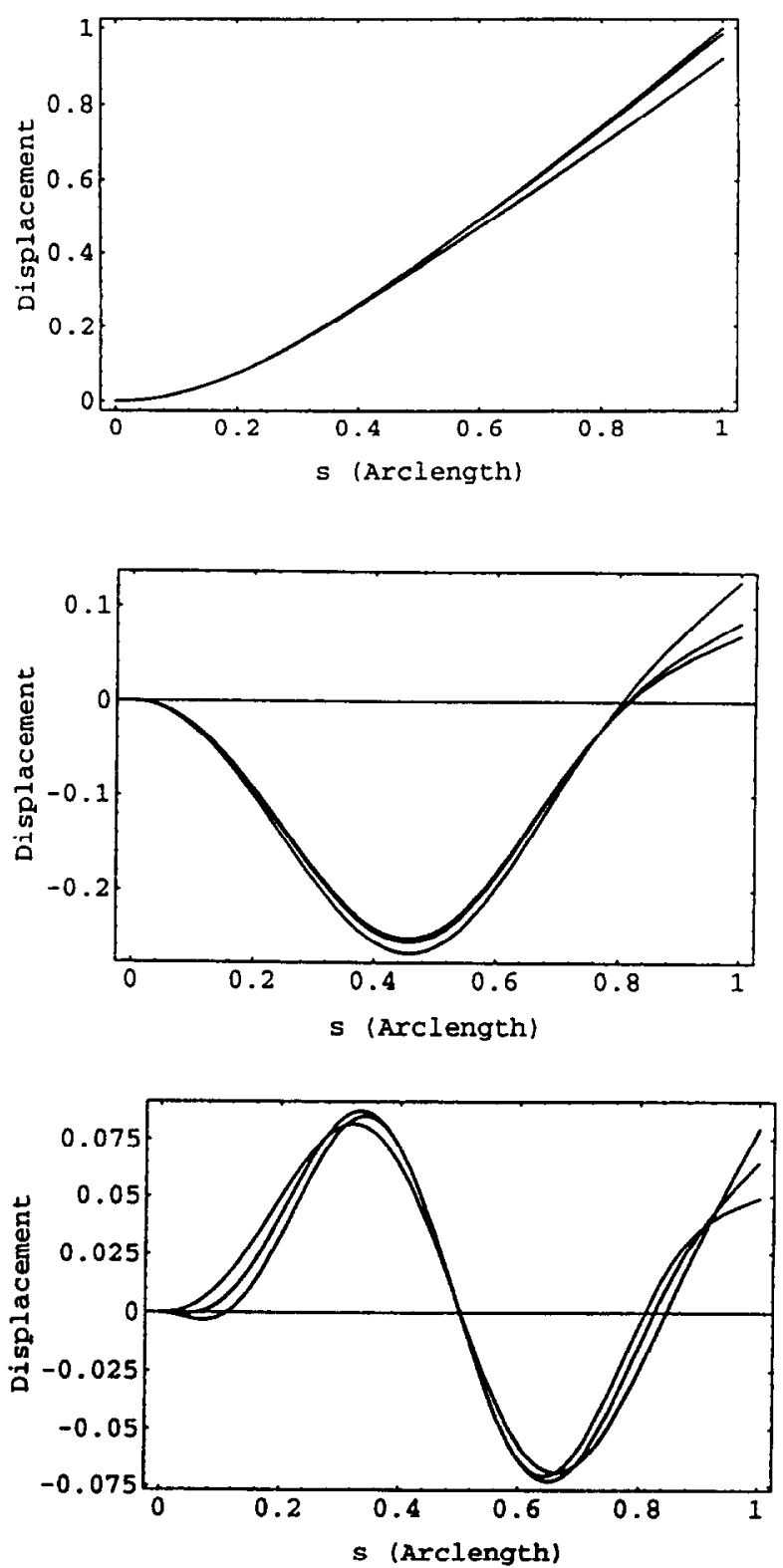

Fig. 12. Typical divergent non-linear normal modes (example 1) : (a) fist mode; (b) second mode; (c) third mode.

work of Shaw and Pierre $(1993,1994)$ for examples and plots of some non-linear normal mode manifolds.

The selection of the reference point $s_{0}$ makes the procedure somewhat cumbersomc. To overcome this shortcoming, the authors (Shaw and Pierre, 1994) have modified the current approach procedure to treat non-linear continuous systems by first applying the usual Galerkin's procedure, with normal modes as trial functions, to discretize the continuous system and obtain a set of ordinary differential equations. This set of ordinary differential equations is then studied by the non-linear normal mode method for discrete systems (Shaw and Pierre, 1993). This approach can improve the current solution procedure and avoid several complications, e.g., the selection of $s_{0}$ and the convergence test of the series expansions for $U$ and $V$ [eqn (13)].

Finally, it should be pointed out that the method for determining non-linear normal modes developed here differs in a fundamental way from previous methods used for continuous systems (see Benamar et al., 1991 ; Bennouna and White, 1984; Szemplinska, 

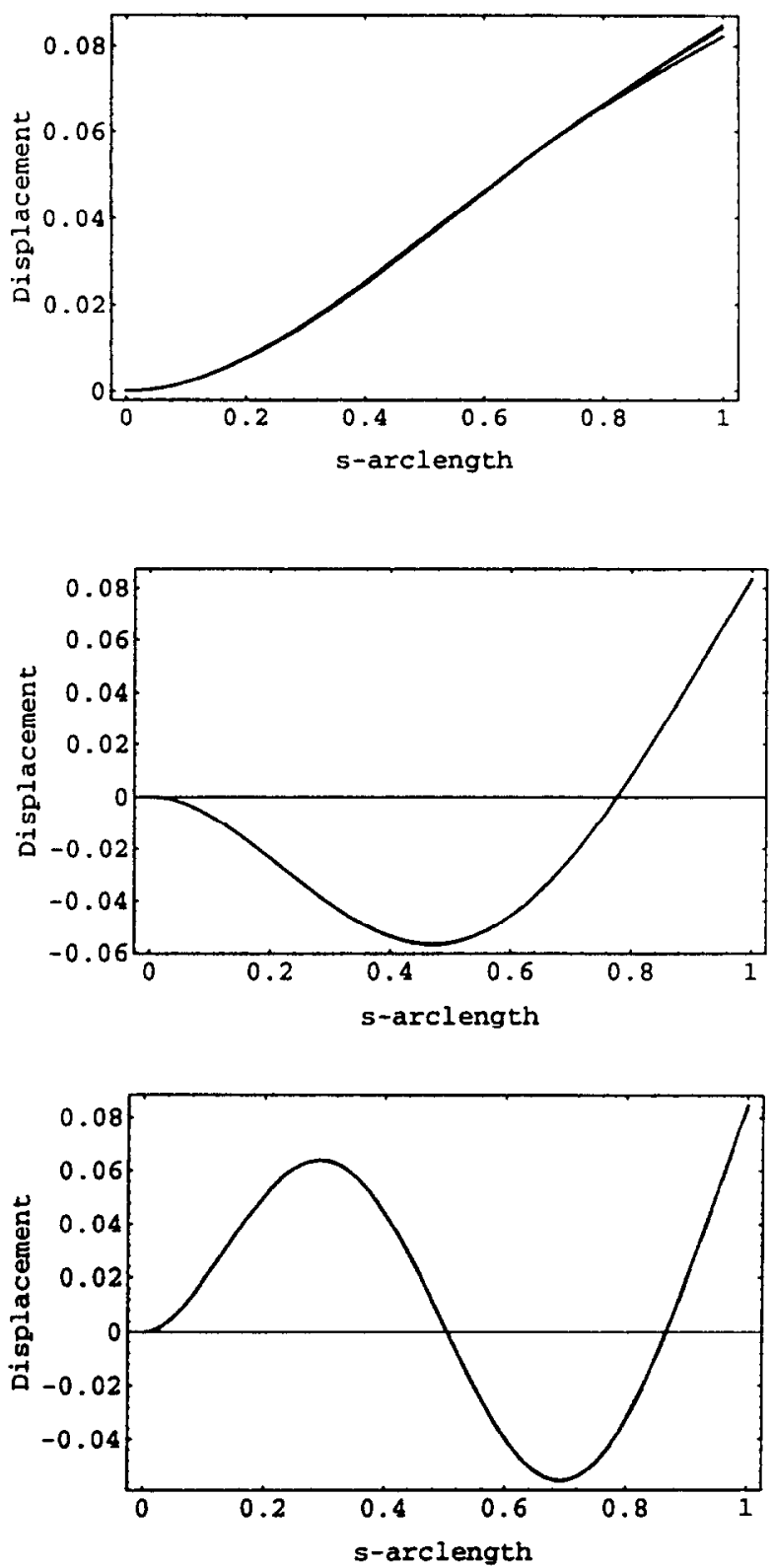

Fig. 13. Typical convergent non-linear normal modes (example 2) : (a) first mode ; (b) second mode ; (c) third mode.

1990). Previous methods separate temporal and spatial behavior at the outset, typically by eliminating the time dependence using harmonic balance and then solving a non-linear eigenvalue problem by eigenfunction expansions. This restriction requires that the beam shape, that is, the relative contribution of each linear mode, be dependent on the peak amplitude of vibration, but fixed as a function of time for a given motion. The present method has no such restriction, since the basic formulation [eqn (5)] and its asymptotic implementation [eqn (13)] allow the shape to be altered during a given motion. This is a lesser restriction and more natural formulation and results in non-linear mode shapes which differ from those obtained using previous methods. The fact that both approaches yield identical results for the estimated frequencies of non-linear oscillation to leading non-linear order is not surprising - this is a variation of Rayleigh's quotient which states that $O(\varepsilon)$ errors in mode shape estimates lead to error at $O\left(\varepsilon^{2}\right)$ in natural frequencies. This observation also implies that higher-order frequency approximations will differ. The source of this 

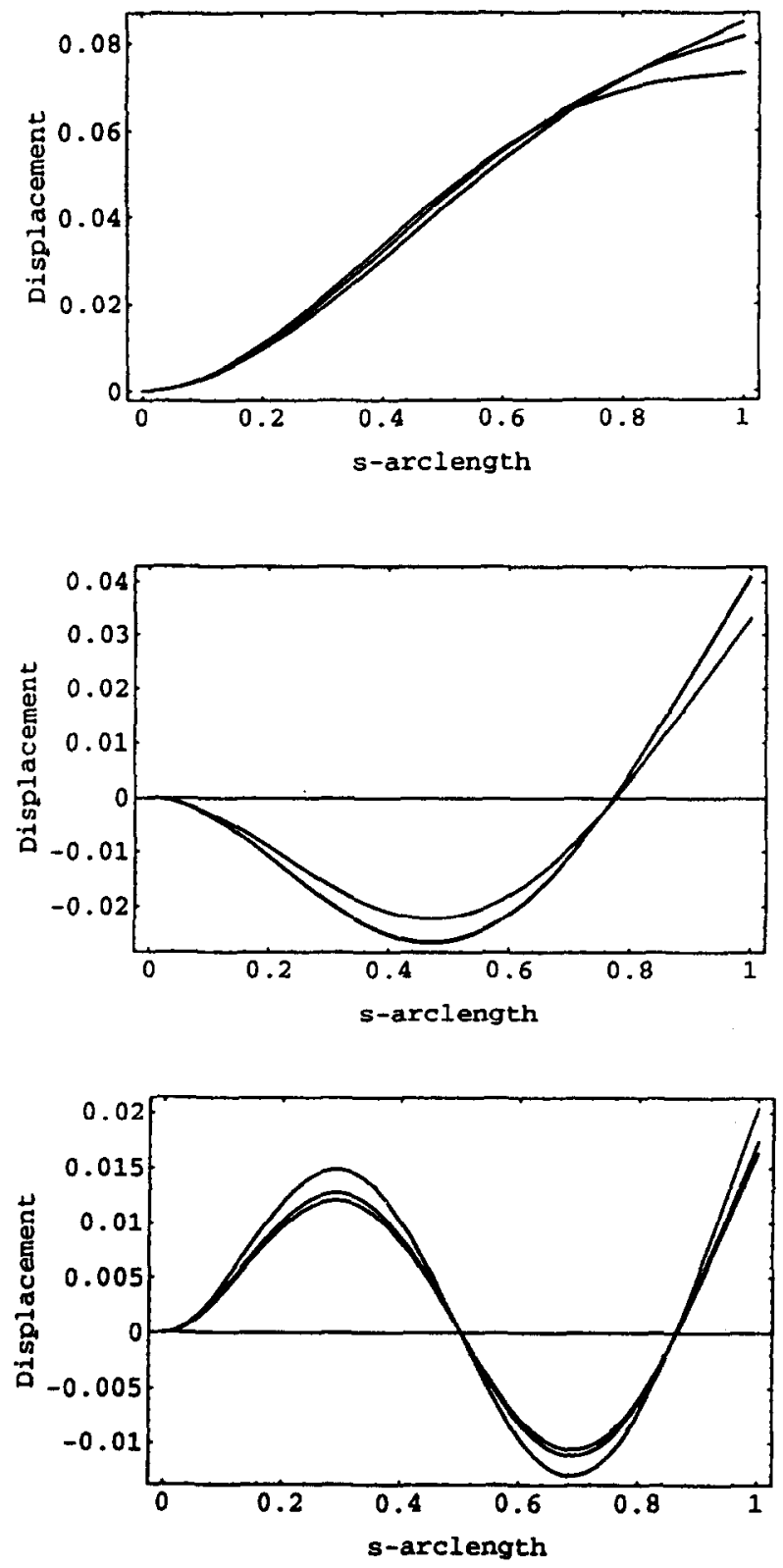

Fig. 14. Typical divergent non-linear normal modes (example 2) : (a) first mode; (b) second mode ; (c) third mode.

difference in the following: The present method does not require any restriction on the nature of the normal mode motion, other than it be that from a second-order autonomous system. This motion is determined from the modal oscillators after the mode shapes have been determined.

Since the invariant manifold approach is quite general and is based on sound principles of dynamical systems, it yields the correct description of non-linear normal modes.

Acknowledgement - This work was supported in parts by grants from NSF and ARO.

\section{REFERENCES}

Benamar, R., Bennouna, M. M. K. and White, R. G. (1991). The effects of large vibration amplitudes on the mode shapes and natural frequencies of thin elastic structures, part I: simply supported and clamped-clamped beams. J. Sound Vibr. 149, 179-195. 
Bennouna, M. M. K. and White, R. G. (1984). The effects of large vibration amplitudes on the fundamental mode shape of a clamped-clamped uniform beam. J. Sound Vibr. 96, 309-331.

Carr, J. (1981). Application of Centre Manifold Theory. Springer, Berlin.

Courant, R. and Hilbert, D. (1961). Methods of Mathematical Physics, Vol. I. Interseience, New York.

Crespo da Silva, M. R. M. and Glynn, C. C. (1978). Nonlinear flexible-flexible-torsional dynamies of inextensional beams. I-equations of motion. J. Struct. Mech. 6, 437-448.

Dowell, E. H. (1984). On asymptotic approximations to beam mode shapes. J. appl. Mech. 51, 439.

Hodges, D. W. (1984). Proper definition of curvature in nonlinear beam kinematics. AIAA J/ 22, 1825-1827.

Gu, K. and Tongue, B. H. (1987). A method to improve the modal convergence for structures with external forcing. J. appl. Mech. 54, $904-909$.

Rand, R. H. (1974). A direct method for non-linear normal modes. Int. J. Non-linear Mech. 9, 363-368.

Rosenberg, R. M. (1966). On non-linear vibrations of systems with many degrees of freedom. Adr. appl. Mech. 9, 155-242.

Pieme, C. and Shaw, S. W. (1991). Mode localization due to symmetr y-breaking nonlinearities. Int. J. Bifurcation and Chaos 1, 471-475.

Shaw, S. W. and Pierre, C. (1992). On nonlinear normal modes. ASME Annual Meeting, Nonlinear Vibrations DE-50, 16

Shaw, S. W. and Pierre, C. (1993). Normal modes for non-linear vibratory systems. J. Sound Vibr. 164, 85-124.

Shaw, S. W. and Pierre, C. (1994). Normal modes of vibration for non-linear continuous systems. J. Sound Vibr. $169,319-348$

Szemplinska, W. (1990). The Behaviour of Non-linear Vibrating Systems, Vol. 2. Kluwer, Dordrecht.

Vakakis, A. (1990). Analysis and identification of linear and non-linear normal modes in vibrating systems. Ph.D. thesis, Caltech, Pasadena, CA. 